\title{
MEANING IN CLASSICAL MATHEMATICS: IS IT AT ODDS WITH INTUITIONISM?
}

\author{
KARIN USADI KATZ AND MIKHAIL G. KATZ*
}

Date: October 26, 2011.

2000 Mathematics Subject Classification. 01A85; Secondary 26E35, 03A05, 97A20, 97C30 .

Key words and phrases. axiom of choice; Bishop; Brouwer; classical logic; constructivism; foundational paradoxes; Goodman-Myhill theorem; Hawking-Penrose singularity theorem; Heyting; hyperreals; indispensability thesis; infinitesimal; intuitionistic logic; Keisler; Kronecker; law of excluded middle; non-standard analysis; numerical meaning; phlogiston; proof by contradiction; Robinson; variational principle; MSC Codes: 01A85; 26E35, 03A05, 97A20, 97C30.

${ }^{*}$ Supported by the Israel Science Foundation grant 1294/06. 
AbStRact. We examine the classical/intuitionist divide, and how it reflects on modern theories of infinitesimals. When leading intuitionist Heyting announced that "the creation of non-standard analysis is a standard model of important mathematical research", he was fully aware that he was breaking ranks with Brouwer. Was Errett Bishop faithful to either Kronecker or Brouwer? Through a comparative textual analysis of three of Bishop's texts, we analyze the ideological and/or pedagogical nature of his objections to infinitesimals à la Robinson. Bishop's famous "debasement" comment at the 1974 Boston workshop, published as part of his Crisis lecture, in reality was never uttered in front of an audience. We compare the realist and the anti-realist intuitionist narratives, and analyze the views of Dummett, Pourciau, Richman, Shapiro, and Tennant. Variational principles are important physical applications, currently lacking a constructive framework. We examine the case of the Hawking-Penrose singularity theorem, already analyzed by Hellman in the context of the Quine-Putnam indispensability thesis.

Résumé: Nous analysons le clivage classique/intuitionniste, et la façon dont il est reflété dans les théories modernes des infiniment petits. Losque l'intuitionniste Heyting annonça que "la création de l'analyse non-standard est un modèle standard de recherche mathématique importante", il fut pleinement conscient du fait qu'il rompait les rangs avec Brouwer. Errett Bishop fut-il fidèle soit à Kronecker soit à Brouwer? Par le biais d'une étude comparative de trois textes de Bishop, nous analysons la nature idéologique et/ou pédagogique de ses objections aux infinitésimaux à la Robinson. La célèbre remarque de Bishop concernant le "debasement", au colloque de Boston en 1974, et publiée dans le cadre de son texte La Crise etc., en réalité ne fut jamais énoncée devant un public. Nous comparons les narratives realiste et anti-realiste intuitionnistes, et analysons les opinions de Dummett, Pourciau, Richman, Shapiro, et Tennant. Les principes variationnels constituent des applications physiques importantes, et qui manquent actuellement de cadre constructif. Nous examinons le cas des théorèmes sur les singularités (de Hawking et Penrose), déjà analysé par Hellman dans le contexte de la thèse de l'indispensabilité de Quine-Putnam.

\section{Contents}

\section{Introduction}

1.1. Cognitive bias

1.2. Birkhoff's opposing schools

1.3. Where Weyl, Crowe, and Heyting agree 
2. A crash course in intuitionism

2.1. The genesis of the constructive project in Kronecker 10

2.2. Kolmogorov on paradoxes 11

2.3. Law of excluded middle and constructive quantifiers $\quad 12$

2.4. Bishop and Brouwerian countexamples 16

3. A kinder, gentler constructivism 16

3.1. The debasement and obfuscation charges $\quad 16$

3.2. Halmos's translation 20

3.3. Why was non-standard analysis the target? 21

3.4. Galley proof "debasement" 23

3.5. A hypothesis and its refutation 24

3.6. Further background on refuted hypothesis 29

4. De-basing in constructivism 33

4.1. Verbal equality 33

4.2. Goodman-Myhill's $1 \frac{1}{2} \pm \frac{1}{2}$ sets and "full" choice 35

4.3. Is Bishopian constructivism compatible with classical logic? 36

5. Are there two constructivisms? 37

5.1. Numerical constructivism or anti-LEM constructivism? 37

5.2. Hilbert and post-LEM meaning 39

5.3. How could an intuitionist accept the hyperreals? 43

6. The fervor of Bishopian constructivism 44

6.1. Pourciau on phlogiston 44

6.2. A constructive glossary 48

6.3. Insurrection according to Michael 51

6.4. Insurrection according to Errett 53

6.5. Richman on large cardinals 54

7. Is constructive mathematics part of classical mathematics? 57

7.1. From Cantor and Frege to Brouwer $\quad 57$

7.2. Heyting's good right 58

7.3. Heyting's Address to Professor Robinson 61

8. Constructivism, physics, and the real world 63

8.1. Bishop's view 63

8.2. Hellman on indispensability 65

8.3. Novikov's perspective 68

8.4. The Hawking-Penrose theorem 68

9. Epilogue $\quad 72$

Appendix A. Rival continua $\quad 72$

Acknowledgments $\quad 76$

References 


\section{IntroduCtion}

1.1. Cognitive bias. The study of cognitive bias has its historical roots in a classification proposed by Francis Bacon (1561 - 1626) of what he called the idola (a Latin plural) of several kinds. He described these as things which obstructed the path of correct scientific reasoning. Of particular interest to us are his Idola fori (Illusions of the Marketplace): due to confusions in the use of language and taking some words in science to have a different meaning than their common usage; and Idola theatri (Illusions of the Theater): the following of academic dogma and not asking questions about the world (see Bacon [11]).

Completeness, continuity, continuum, Dedekind "gaps": these are terms whose common meaning is frequently conflated with their technical meaning. In our experience, explaining infinitesimal-enriched extensions of the reals to a professional mathematician typically elicits a puzzled reaction of the following sort: "But how can you extend the real numbers? Aren't they already complete by virtue of having filled in all the gaps already?" The academic dogma concerning the uniqueness of the continuum defined necessarily as the complete Archimedean ordered field in the context of ZFC has recently been challenged in the pages of Intellectica, where numerous conceptions of the continuum were presented, ranging from S. Feferman's predicative conception of the continuum [68] to J. Bell's conception in terms of an intuitionistic topos [17] following Lawvere [125].

To illustrate the variety of possible conceptions of the continuum, note that traditionally, mathematicians have considered at least two different types of continua. These are Archimedean continua, or Acontinua for short, and infinitesimal-enriched (Bernoulli) continua, or B-continua for short. Neither an A-continuum nor a B-continuum corresponds to a unique mathematical structure (see Table 1.1). Thus, we have at least two distinct implementations of an A-continuum:

- the real numbers in the context of classical logic (incorporating the law of excluded middle);

- Brouwer's real continuum built from "free-choice sequences", in the context of intuitionistic logic.

John L. Bell describes a distinction within the class of infinitesimalenriched B-continua, in the following terms. Historically, there were two main approaches to such an enriched continuum, one by Leibniz, and one by B. Nieuwentijdt. The latter favored nilpotent (nilsquare) infinitesimals. J. Bell notes: 


\begin{tabular}{|l||l|l|}
\hline & Archimedean & Bernoullian \\
\hline \hline classical & $\begin{array}{l}\text { Stevin's } \\
\text { continuum } 3\end{array}$ & $\begin{array}{l}\text { Robinson's } \\
\text { continuum } 1\end{array}$ \\
\hline intuitionistic & $\begin{array}{l}\text { Brouwer's } \\
\text { continuum }\end{array}$ & $\begin{array}{l}\text { Lawvere's } \\
\text { continuum }\end{array}$ \\
\hline
\end{tabular}

TABLE 1.1. Varieties of continua, mapped out according to a pair of binary parameters: classical/intuitionistic and Archimedean/Bernoullian.

Leibnizian infinitesimals (differentials) are realized in [A. Robinson's] nonstandard analysis 1 and nilsquare infinitesimals in [Lawvere's] smooth infinitesimal analysis [15, 16].

The latter theory relies on intuitionistic logic 2 A more recent implementation of an infinitesimal-enriched continuum has been developed by P. Giordano, see [76, 77, 78], combining elements of both a classsical and an intuitionistic continuum. The familiar real continuum is rooted in the 16th century work of Simon Stevin 3

1.2. Birkhoff's opposing schools. J. Dauben reports that Garrett Birkhoff made the following statement at a Workshop on the evolution of modern mathematics in 1974:

During the past twenty years, significant contributions to the foundations of mathematics have been made by two opposing schools [51, p. 132].

Birkhoff proceeded to identify the opposing schools as Abraham Robinson's non-standard approach, and Errett Bishop's constructive approach, which, according to Birkhoff,

attempts to reinterpret Brouwer's "intuitionism" in terms of concepts of 'constructive analysis'.

S. Warschawski referred to non-standard analysis as

\footnotetext{
${ }^{1}$ More precisely, the Hewitt-Łoś-Robinson continuum; see footnote 63 .

${ }^{2}$ Lawvere's infinitesimals rely on intuitionistic logic. Thus, Lawvere's system is incompatible with classical logic, see J. Bell [16]. The situation with Bishop's constructive mathematics is more complex; see Subsection 4.3 .

${ }^{3}$ See A. Malet [130, J. Naets [136, Katz \& Katz 113.
} 
a theory that has been described as diametrically opposed to constructivism [185, p. 37].

In 1999, a conference [158] sought to reunite the antipodes (another term for our pair of opposing schools). D. Ross [155, p. 494] evokes a conceptual gulf between the two approaches.

Robinson's approach to infinitesimals has a coherent methodology in the framework of the Zermelo-Fraenke] set theory with the axiom of choice, in the context of classical logic (incorporating the law of excluded middle).

The situation with intuitionism/constructivism is more nebulous. Within this school, we will distinguish two significantly different approaches. One of the approaches can arguably be associated with A. Heyting's views [101, 102], as well as with Bishop's views [25] as expressed in 1975. The other approach can be associated with Bishop's views [26] as expressed in 1977, as well as with M. Beeson [13] and M. Dummett [60] 5 A related dichotomy of liberal versus radical constructivism was analyzed by G. Hellman [95. Rather than borrowing terms from political science, we will exploit terms that are somewhat more self-explanatory in a mathematical context, namely the view of constructive mathematics as a companion versus an alternative to classical mathematics.

The infinitesimal, affectionately nicknamed the cholera bacillus of mathematics by G. Cantor 6 has mutated into a bone of contention between our pair of antipodes, a bone constricting the constructivist throat of our students' aspirations, according to some; and the elegant answer to Leibniz's dream, 7 according to others.

The possibility of a peaceful coexistence between our pair of antipodes is not an idle dream, but rather a matter of historical record. In 1954, Robinson gave a lecture at a symposium on the "Mathematical interpretation of formal systems" chaired by A. Heyting, see [52, p. 254]. In 1961, Robinson made public his new idea of non-standard models for analysis, and "communicated this almost immediately to A[.] Heyting" [52, p. 259]. Robinson's first paper on the subject was subsequently published in Proceedings of the Netherlands Royal Academy of Sciences [147]. An appreciation of Heyting's work appears in Robinson's 1968 text:

\footnotetext{
${ }^{4}$ See footnote 36 on Fraenkel's comments on infinitesimals.

${ }^{5}$ Dummett's views will be examined in Section 6.3.

${ }^{6}$ See Meschkowski [133, p. 505] and Dauben [50, p. 353], [51, p. 124].

7 Note that Schubring [157, p. 170, 173, 187] attributes the first systematic use of infinitesimals as a foundational concept, to Johann Bernoulli (rather than Leibniz).
} 
Among those who have shown a consistently constructive attitude we may mention Heyting.... [149, p. 921].

In 1973, Heyting praised non-standard analysis as "a standard model of important mathematical research" [102, p. 136].8

Meanwhile, S. Shapiro unveils an allegedly irreducible clash between classical mathematics, on the one hand, and intuitionism as expressed through Heyting's semantics (see [160, p. 60]), on the other 9

From the viewpoint of classical mathematics, what is a reasonable scope of the constructivist critique of the foundations? We will describe the proposed dichotomy within constructivism, and argue that only one of the approaches would be potentially amenable to a possible reunification (or at least a state of non-belligerence) alluded to in the title of the conference [158].

While commenting on the "role of non-standard analysis in mathematics", P. Halmos writes:

For some other[...mathematicians], who are against it (for instance Errett Bishop), it's an equally emotional issue [89, p. 204].

What was it specifically about non-standard analysis, among other fields of classical mathematics, that prompted Bishop's opposition? We will analyze this issue in Subsections 3.1 and 3.3 .

Does intuitionism have an Achilles' heel? Without attempting to provide a definitive answer, we list potential vulnerabilities in Table 1.2 , arranged by field.

A recent analysis of the Classical vs Intuitionistic divide by D. Westerståhl posits that

The typical intuitionist takes truth to be what philosophers call an epistemic notion: roughly, something is true if it can be proved. [188, p. 177].

Such a summary would be agreed upon by many intuitionists. But then Westerståhl concludes:

This puts computation at center stage: (intuitionistic) proofs are computations, or directions for finding computations [188, p. 177].

Westerståhl's reformulation of constructively meaningful mathematics in terms of "computations" betrays an unmistakable influence of

\footnotetext{
${ }^{8}$ Heyting's appreciation of Robinson's theory is analyzed in more detail in Subsection 7.3 .

${ }^{9}$ Shapiro's views will be analyzed in Subsection 7.2
} 


\begin{tabular}{|l||l|l|}
\hline field & issues & $\begin{array}{l}\text { discussed } \\
\text { in: }\end{array}$ \\
\hline \hline Mathematics & $\begin{array}{l}\text { Arguments in favor of coherence of } \\
\text { post-LEM numerical meaning }\end{array}$ & Section 5.2 \\
\hline Physics & $\begin{array}{l}\text { Variational principles, Hawking- } \\
\text { Penrose theorem, Hilbert's Lagrangian }\end{array}$ & Section 8.4 \\
\hline Philosophy & $\begin{array}{l}\text { Diverging insurrectional narratives: } \\
\text { realist and anti-realist }\end{array}$ & Section 6.3 \\
\hline History & $\begin{array}{l}\text { Brouwer's intellectual debt to Frege's } \\
\text { revolution and Cantor's revolution }\end{array}$ & Section 6.3 \\
\hline Defection & $\begin{array}{l}\text { Weyl, Heyting, Bridges arguably side } \\
\text { with a companion view }\end{array}$ & $\begin{array}{l}\text { Sections 7.2, } \\
\text { 7.3, 8, 6.5 }\end{array}$ \\
\hline
\end{tabular}

TABLE 1.2. Radical Intuitionism: key issues

Bishop's perspective, which tends to conflate meaning with computational meaning, as we will analyze below. Similarly, on the same page it emerges that, to Westerståhl (following Dummett), classical mathematics means Platonism. No wonder that his evaluation of the possibility of reaching a mutual understanding

is mostly negative: a basic asymmetry as to one side's ability to achieve understanding of what the other is up to will remain [188, p. 177].

Meanwhile, our own assessment is mostly positive, relying on a more nuanced presentation of both sides of what we hold to be a bridgeable divide.

1.3. Where Weyl, Crowe, and Heyting agree. We conclude this introduction by quoting Hermann Weyl:

It is pretty clear that our theory of the physical world is not a description of the phenomena as we perceive them, but [rather] is a bold symbolic construction. However, one may be surprised to learn that even mathematics shares this character [191, p. 553]. 
No single formalism, whether classical or intuitionistic, represents "a description of the [mathematical] phenomena", to borrow Weyl's phrase. This sentiment is echoed by M. Crowe 10

creative mathematicians have repeatedly encountered situations that are not resolvable by logic, that are no less dependent on brilliance of mind and an ability to see beyond logic, than those that have been faced by physicists. One example would be the situation faced by the pioneers of the calculus, who pushed forward despite being surrounded by inconsistencies and counterintuitive deductions [44], [45, p. 316] [emphasis addedauthors].

In reference to classical mathematics, a similar thought is expressed by the intuitionist Heyting, who described it as a "curious mixture of formal reasoning and more or less vague intuitions".

We analyze the sources of constructive aspirations in Kronecker's vision in Subsection 2.1, In Subsection 2.2, we give a gentle introduction to the intuitionistic challenge, following A. N. Kolmogorov. In Subsection 2.3, we give some examples of what it would mean to eliminate the reliance on the law of excluded middle. In Subsection 2.4, we compare Brouwerian and Bishopian mathematics.

In Subsection 3.1 we document some specific criticisms voiced by Bishop. In Subsection [3.2, we examine the dual aspects of Halmos's relation to Robinson's theory. In Subsection 3.3, we analyze the nature of the challenge to Bishopian constructivism posed by a modern theory of infinitesimals. The story behind Bishop's "debasement" comment is told in Subsection 3.4. Subsections 3.5 and 3.6 analyze the foundational nature of Bishop's criticisms.

The shifting ground of constructivist definitions is reviewed in Section 4.1. The meaning of "finite" in the context of the Goodman-Myhill theorem is reviewed in Section 4.2. Section 4.3 examines the finessing of Brouwerian counterexamples in Bishopian constructivism.

A dichotomy of two distinct approaches within constructivism is proposed in Subsection 5.1. Section 5.2 introduces a dichotomy of preLEM and post-LEM numerical meaning. Subsection 5.3 examines a philosophical outlook that can permit an intuitionist to accept both types of meaning.

Section 6 deals with the fervor of Bishopian constructivism. Subsection 6.1 examines the views of B. Pourciau. A glossary of Bishopian constructivism appears in Subsection 6.2, followed by an analysis of a

\footnotetext{
${ }^{10}$ See footnote 122
} 
pair of insurrectional narratives in Subsections 6.3 and 6.4. Section 6.5 deals with the views of constructivist F. Richman.

Section 7 examines the issue of whether constructive mathematics is part of classical mathematics. Subsection 7.1 examines the logical developments, including Frege's relational logic, in the 19th century that created the conditions that made intuitionistic revolt possible. We focus on Heyting's intuitionism in Subsection 7.2, where we also comment on the views of Shapiro and Tennant (the views of the latter are also examined in Subsection 8.4). Heyting's appreciation of nonstandard analysis is examined in Subsection 7.3 .

Section 8 deals with a challenge to constructivism arising from natural science. Subsection 8.1 deals with Bishop's own view of the applicability of mathematics in the natural sciences. Section 8.2 deals with G. Hellman's indispensability thesis approach. S.P. Novikov's perspective is discussed in Subsection 8.3. The Hawking-Penrose theorem and its foundational ramifications are analyzed in Subsection 8.4.

\section{A CRASH COURSE IN INTUITIONISM}

We present some preliminary remarks on the intuitionist project from Kronecker to Brouwer and beyond. More technical aspects of constructivism are dealt with in Section 4 .

2.1. The genesis of the constructive project in Kronecker. While constructivist aspirations can be detected already in a predilection for the rationals (at the expense of broader number systems) on the part of mathematicians both ancient and modern, the roots of 20th century constructivism are surely to be sought in L. Kronecker. J. Boniface and N. Schappacher recently edited an important unpublished essay of Kronecker's. Here Kronecker envisions a 3-part subdivision of mathematics as follows:

la mécanique, qui opère avec la notion de temps, la géométrie, qui étudie les relations spatiales indépendantes du temps, et la mathématique dite pure, dans laquelle n'interviennent ni le temps ni l'espace, et que je veux appeler 'arithmétique 11 [30, p. 211].

Thus, mathematics is subdivided by Kronecker into mechanics (which apparently corresponds to mathematical physics), geometry, and arithmetic. Kronecker proceeds to state the scope of his constructivist project of the reduction of mathematics to the integers, in the following terms:

\footnotetext{
${ }^{11}$ These comments appear on pp. $10-11$ in Kronecker's manuscript.
} 
Il souligne (p. 15) que "tout ce qui n'appartient pas à la mécanique et à la géométrie, et que je veux rassembler sous l'intitulé d'arithmétique, devrait être effectivement arithmétisé" [30, p. 211].

Thus, the only branch of mathematics that is to be arithmetized (i.e., converted into a discourse about the natural numbers), according to Kronecker, is that which falls outside the domain of both geometry and mathematical physics. The implication is that geometry need not, or could not, be so arithmetized, in Kronecker's opinion. Kronecker provides a reasonable limitation to the scope of his constructivist project. Boniface and Schappacher further note:

'Arithmétiser' consiste, pour Kronecker, non seulement à réduire les objets mathématiques aux entiers positifs, mais aussi à n'utiliser que des méthodes arithmétiques [30, p. 211] ... Les concepts de nombre négatif et de nombre fractionnaire étant évités en tant que concepts fondamentaux de l'arithmétique, celui de nombre irrationnel le sera a fortiori. L'irrationalité est un concept géométrique et doit, selon Kronecker, rester dans le domaine géométrique [30, p. 213].

In short, geometry (including irrational numbers) falls outside the scope of Kronecker's constructivist project. In his moderation of scope, Kronecker was not followed by all of his would-be disciples.

2.2. Kolmogorov on paradoxes. Eighty years ago, A. Kolmogorov described the emergence of both Hilbert's formalism and Brouwer's intuitionism, as

a reaction against the set-theoretic conception of mathematics [121, p. 380].

Kolmogorov speaks of great difficulties and even contradictions (ibid. p. 382) caused by set-theoretic mathematics, citing Russell's paradox as an example (ibid. p. 383). As to Zermelo's axiom of choice, he writes:

it came to a hopeless collision with the idea that mathematic[al] existence should be based on a[n appropriate] construction [...] objects whose existence is postulated by this axiom appeared to be not only useless but sometimes destructive to the simplicity and [rigorousness] of crucial mathematical theories.

The recent republication [121] of Kolmogorov's text was annotated by V. Uspenskiy. In his note 15 on page 387, Uspenskiy illustrates the 
above comment in terms of the famous Banach-Tarski paradox (see [183]).

In short, a significant mathematical minority has felt that the paradoxes of set theory have sapped the faith in both set-theoretic foundations and classical logic.

How is one to view the classical axiom of choice and its counterintuitive consequences such as the Banach-Tarski paradox? There could be at least two distinct approaches:

(1) one can maintain that "infinite sets behave differently from finite sets" (and in particular have properties that appear to be paradoxical when compared to finite sets); alternatively,

(2) some of the basic assumptions of set-theoric foundations and classical logic need to be re-examined.

A classically trained mathematician typically follows the first approach. Meanwhile, an intuitionist would follow the second approach, and would ask the classical mathematician: "Certainly, infinite sets behave differently from finite sets. But can you exhibit a single such paradoxical infinite 'set' explicitly? The only way of accessing such 'sets' is by invoking the classical axiom of choice and the law of excluded middle. Since such 'sets' tend to contradict our intuition of physical space, the reasonable alternative is to reject both the 'sets' themselves, and whatever dubious axioms and procedures are responsible for propelling them into existence in the first place."12

The procedures in question are detailed in the next section.

2.3. Law of excluded middle and constructive quantifiers. A gentle introduction to intuitionism would necessarily seek to clarify the law of excluded middle by providing suitable examples (see below). The meta-mathematical language of mainstream mathematics relies on what is generally referred to as classical logic, which we will briefly denote

$$
\text { ClLo, }
$$

incorporating the Law of Excluded Middle (LEM). The law of excluded middle is the key ingredient in a proof by contradiction, as Example 2.1 below illustrates. H. Billinge writes that E. Bishop

seems to take it that common sense would balk at allowing the assertion of 'there exists an $x$ such that $P(x)$ ' on the flimsy basis of having shown that a contradiction

\footnotetext{
${ }^{12}$ All constructivists reject the sets involved in paradoxical decompositions à la Banach-Tarski. Infinite sets (actual infinity) are acceptable to Bishop's followers, see footnote 24. but not to Dummett's followers, see footnote 104.
} 
can be derived from assuming that there is no $x$ such that $[\ldots] P(x)[21$, p. 184].

She concludes that

the only reason that we ordinarily accept such a derivation as evidence for such an existence claim must be that we take it that we are dealing with a finite domain and hence that we would be able to find the $x$ in question by examination [i.e., "by inspection"] 13

We will illustrate the difference between the classical and the constructive approach by means of some typical examples.

Example 2.1. Irrationality of $x$ is defined constructively in terms of concrete separation between $x$ and each rational $\frac{m}{n}$ (separation being expressed in terms of the denominator $n$ ). The classical proof of the irrationality of $\sqrt{2}$ is a proof by contradiction. Namely, we assume a hypothesized equality $\sqrt{2}=\frac{m}{n}$, square both sides, examine the parity of the powers of 2 , and arrive at a contradiction. At this stage, irrationality is considered to have been proved, in ClLo 14

Alternative, numerically meaningful (see glossary in Subsection 6.2), arguments for irrationality exist. Thus, without exploiting the hypothetical equality $\sqrt{2}=\frac{m}{n}$, one can exhibit positive lower bounds for the

\footnotetext{
${ }^{13}$ Of course, no computer can examine, or inspect, a collection containing, say, $10^{100}$ elements. Billinge's comments should not be interpreted as an endorsement of a strict finitism (certainly not espoused by Bishop), but rather as a way of introducing an intuitionistic viewpoint to a classical reader unfamiliar with this circle of ideas.

${ }^{14}$ The classical proof that $\sqrt{2}$ is not rational is acceptable in intuitionistic logic. To pass from this to the claim of its irrationality as defined above, requires LEM, or more precisely the law of trichotomy: $(x<0) \vee(x=0) \vee(x>0)$ (see Subsection 4.3 for more details on the latter). Our purpose here is not to present an exhaustive treament of the intuitionistic perspective on irrationality, but rather to give an accessible illustration of what might be considered a numerically meaningful argument, as opposed to an indirect proof (by contradiction).
} 
difference $\left|\sqrt{2}-\frac{m}{n}\right|$ in terms of the denominator $n$, resulting in a constructively adequate proof of irrationality 15 A more detailed discussion of this example, by E. Bishop, may be found at [27, p. 18].

Brouwer's intuitionism, as well as most forms of constructivism, rely on intuitionistic logic, which we will briefly denote

$$
\text { InLo, }
$$

characterized by the rejection of LEM. The relation can be represented graphically as follows: 16

$$
\mathrm{ClLo}-\mathrm{LEM} \approx \mathrm{InLo} .
$$

Similarly, constructive quantifiers are different from the classical ones, in that their domain of discourse is reduced to constructive entities 17 Constructivism adopts a verificational interpretation of the quantifiers. In this context, it is relevant to mention that the (sub)finite axiom of choice would imply LEM, see Section 4.2. To emphasize the distinction between two types of quantifiers, one could incorporate a mention of ClLo (respectively, InLo) as part of the quantifier notation, as the following example illustrates.

Example 2.2. Consider the following special case of the axiom of choice. Let $2^{\mathbb{R}}$ be the set of sets of reals, and $2^{2^{\mathbb{R}}}$, the set of sets of sets of reals. Let

$$
2^{2^{\mathbb{R}}}
$$

\footnotetext{
${ }^{15}$ For each rational $m / n$, the integer $2 n^{2}$ is divisible by an odd power of 2 , while $m^{2}$ is divisible by an even power of 2 . Hence $\left|2 n^{2}-m^{2}\right| \geq 1$ (here we have applied LEM to an effectively decidable predicate over $\mathbb{Z}$, or more precisely the law of trichotomy). Since the decimal expansion of $\sqrt{2}$ starts with $1.41 \ldots$, we may assume $\frac{m}{n} \leq 1.5$. It follows that$$
\left|\sqrt{2}-\frac{m}{n}\right|=\frac{\left|2 n^{2}-m^{2}\right|}{n^{2}\left(\sqrt{2}+\frac{m}{n}\right)} \geq \frac{1}{n^{2}\left(\sqrt{2}+\frac{m}{n}\right)} \geq \frac{1}{3 n^{2}},
$$

yielding a numerically meaningful proof of irrationality.

${ }^{16}$ The approximate equality sign " $\approx$ " reflects the fact that there are many subsystems of classical logic which, when LEM is adjoined to them, yield all of classical logic. Thus, intuitionistic logic is not uniquely characterized by the rejection of LEM. Still, such a rejection is the main theme of intuitionism (see Bishop's comment on the axiom of choice in Subsection 4.2). While it may have been more correct to write InLo + LEM $=$ ClLo, we chose to emphasize the passage from classical logic to intuitionistic logic, rather than vice versa, keeping in mind the dominant modes of logical reasoning of the mathematical public today.

${ }^{17} \mathrm{We}$ are mostly following Bishop's views here; for a discussion of Richman's views, see Subsections 6.5 and 8.1
} 
be the set of sets of mutually disjoint, non-empty sets of reals. In this case, the classical axiom of choice asserts that

$$
\left(\forall_{\mathrm{ClLo}} A \in 2_{\amalg}^{2^{\mathbb{R}}}\right)\left(\exists_{\mathrm{ClLo}} S \in 2^{\mathbb{R}}\right)\left(\forall_{\mathrm{ClLo}} x \in A\right)|x \cap S|=1 .
$$

In the case of a finite set, say

$$
\{0,1\}
$$

the corresponding formula

$$
\left(\forall_{\mathrm{ClLo}} A \in 2_{\amalg}^{2^{\{0,1\}}}\right)\left(\exists_{\mathrm{ClLo}} S \in 2^{\{0,1\}}\right)\left(\forall_{\mathrm{ClLo}} x \in A\right) \quad|x \cap S|=1
$$

can be proved by induction. Moreover, by the Goodman-Myhill theorem (see Section 4.2), formula (2.2) implies LEM 18

An intuitionistic version is obtained by replacing the subscript ClLo by InLo (see (2.1) ) on all quantifiers: 19

$$
\left(\forall_{\text {InLo }} A \in 2_{\amalg}^{2^{\{0,1\}}}\right)\left(\exists_{\text {InLo }} S \in 2^{\{0,1\}}\right)\left(\forall_{\text {InLo }} x \in A\right) \quad|x \cap S|=1
$$

Formula (2.3) applies to constructively finite sets but not to subfinite sets 20 Namely, the domain of the quantifiers is limited to constructively defined sets only. In particular, (2.3) does not prove LEM, and is constructively acceptable 21

The intuitionistic way of thinking represents a major paradigm shift. Many classically-trained mathematicians find it difficult to appreciate either its utility or the well-foundedness of its trademark distrust of proofs by contradiction. Hopefully the examples provided in this section provide a start for such an appreciation.

The following argument is apparently well-known in constructive circles 22 It concerns the claim of the consistency of the foundations of mathematics. The argument in favor of the claim is indirect, relying on a proof by contradiction. It is surprisingly short, and goes as follows. Consider the claim that the foundations of mathematics are consistent. Suppose not. Then there is an inconsistency in the foundations. We get a contradiction, proving the claim 23

\footnotetext{
${ }^{18}$ See footnote 74 for a detailed discussion of this implication.

${ }^{19}$ When switching to intuitionistic quantifiers, it is customary to replace "nonempty sets" by "inhabited sets", so as to avoid a linguistic excluded-third pitfall, see footnote 74

${ }^{20}$ Subfinite sets are discussed in more detail in Subsection 4.2.

${ }^{21}$ Concerning the infinite constructive axiom of choice, see Bishop's remark in Subsection 4.2 .

${ }^{22}$ Communicated by Gabriel Stolzenberg.

${ }^{23}$ At this point the classical mathematician typically bursts out laughing at the proof, while the constructivist may perhaps smile at the classical mathematician.
} 
2.4. Bishop and Brouwerian countexamples. E. Bishop was a leading representative of the tendency described as intuitionist or constructivist 24 Among a variety of intuitionistic schools, Bishopian constructivism has been one of the most influential, both mathematically and philosophically. Bishop's perspective on the Classical/Intuitionistic divide has influenced even would-be "outside" observers. Thus, Westerståhl claims that

[Bishop's] aim was to do constructive mathematics that looked just like ordinary mathematics, not even apparently contradicting any classical theorems [188, p. 185].

However, Brouwerian counterexamples do appear in Bishop's work. This could not be otherwise, since a verificational interpretation of the quantifiers necessarily results in a clash with classical mathematics. As a matter of presentation, the conflict with classical mathematics had been de-emphasized by Bishop. Bishop finesses the issue of Brouwer's theorems (e.g., that every function is continuous) by declaring that he will only deal with uniformly continuous functions to begin with. In Bishopian mathematics, a circle cannot be decomposed into a pair of antipodal sets. A real number $a$ satisfying $\neg((a \leq 0) \vee(a \geq 0))$ yields a counterexample to the extreme value theorem 25

\section{A KINDER, GENTLER CONSTRUCTIVISM}

In Section 2.4, we discussed Bishop's mathematical and philosophical influence. Bishop put such influence to a variety of uses.

3.1. The debasement and obfuscation charges. Bishop's Crisis 26 essay [25] is cast in the form of an imaginary dialog between Brouwer and Hilbert. Bishop narrates a creation story of intuitionism in the form of such an imaginary dialog, where Brouwer completely dominates the exchange. Indeed, Bishop's imaginary Brouwer-Hilbert exchange is dominated by an unspoken assumption that Brouwer is the only one who seeks "meaning", an assumption that his illustrious opponent is never given a chance to challenge.

Hopefully we have succeeded in conveying to our gentle reader, a sense of a constructivist reception of a typical proof by contradiction.

${ }^{24}$ It should be kept in mind, however, that Bishop rejects both Kronecker's finitism (as Bishop freely exploits the actual infinity of the natural numbers) and Brouwer's theory of the continuum, see main text at footnote 60 .

${ }^{25} \mathrm{~A}$ more detailed discussion may be found in Subsection 4.3 .

${ }^{26}$ Apparently a take-off on H. Weyl's own Crisis text 189, see also end of Section 8 . 
Meanwhile, Hilbert's comments in 1919 reveal clearly his attachment to meaning which he refers to as internal necessity:

We are not speaking here of arbitrariness in any sense. Mathematics is not like a game whose tasks are determined by arbitrarily stipulated rules. Rather, it is a conceptual system possessing internal necessity that can only be so and by no means otherwise [104, p. 14] 27

Bishop expressed his views about Robinson's infinitesimals and their use in teaching in a brief paragraph toward the end of his text. Following his discussion of Hilbert's formalist program, Bishop inserted the following text:

A more recent attempt at mathematics by formal finesse is non-standard analysis. I gather that it has met with some degree of success, whether at the expense of giving significantly less meaningful proofs I do not know. My interest in non-standard analysis is that attempts are being made to introduce it into calculus courses. It is difficult to believe that debasement of meaning could be carried so far 28 [25, p. 513-514] [emphasis addedauthors].

Bishop's view of the introduction of Robinson-style infinitesimals in the classroom as no less than a debasement of meaning (see glossary in Subsection 6.2), was duly noted by J. Dauben 29 Bishop's sentiments toward calculus based on Robinson-style infinitesimals stand in sharp contrast with those of his fellow intuitionist A. Heyting, who felt that

[Robinson] connected [an] extremely abstract part of model theory with a theory apparently so far apart as the elementary calculus. In doing so [he] threw new light on the history of the calculus by giving a clear sense to Leibniz' 30 notion of infinitesimals [102, p. 136].

Heyting's views are analyzed in more detail in Subsections 7.2 and 7.3 .

\footnotetext{
${ }^{27}$ Cited in Corry [43. See also footnote 55 ,

${ }^{28}$ The story behind the insertion of this paragraph is told in Subsection 3.4 .

${ }^{29}$ See [49, 51]. While there was apparently a wave of reactions to Bishop's review at the time, surprisingly little of it has made its way into print. Other than Dauben's essays already mentioned, there is Keisler's own brief measured response [115], followed in the same issue of the Notices by some comments by V. Komkov [122, see footnote 49. Komkov's letter was mentioned in a MathSciNet review by P. Smith [162]. Bishop's review was also examined briefly by M. Davis [55, see footnote 34 .

${ }^{30}$ But see footnote 7 for a historical clarification by Schubring.
} 
Bishop anchors his foundational stance in a species of mathematical constructivism in the following terms:

To my mind, it is a major defect of our profession that we refuse to distinguish [...] between integers that are computable and those that are not $[\ldots]$ the distinction between computable and non-computable, or constructive and non-constructive is the source of the most famous disputes in the philosophy of mathematics [25, pp. 507-508].

On page 511, Bishop defines a limited principle of omniscience (LPO) as the supposition that

it is possible to search "a sequence of integers to see whether they all vanish",

and goes on to characterize the dependence on the LPO as a procedure both Brouwer and Bishop himself reject 31 The search for numerical meaning is a goal that can appeal to any mainstream mathematician. This thread will be pursued further in Subsection 5.1.

S. Feferman [66, end of section A of part I] identifies LPO as a special case of the law of excluded middle, see Section 2.3 above.

Given that a typical construction of Robinson's infinitesimals (see Keisler [116, p. 911]) certainly does rely on LPO (but see [139]), Bishop's opposition to such infinitesimals, expressed in a vitriolid 32 review of Keisler's textbook, may have been expected. Indeed, Bishop wrote:

The technical complications introduced by Keisler's approach are of minor importance. The real damage lies in [Keisler's] obfuscation and devitalization of those wonderful ideas [of standard calculus]. No invocation of Newton and Leibniz is going to justify developing calculus using axioms $\mathrm{V}^{*}$ and $\mathrm{VI}^{*}$-on the grounds that

\footnotetext{
${ }^{31}$ S. Feferman explains Bishop's principle as follows: "Bishop criticized both nonconstructive classical mathematics and intuitionism. He called non-constructive mathematics 'a scandal', particularly because of its 'deficiency in numerical meaning'. What he simply meant was that if you say something exists you ought to be able to produce it, and if you say there is a function which does something on the natural numbers then you ought to be able to produce a machine which calculates it out at each number" [66] [emphasis added-authors].

${ }^{32}$ Historians of mathematics have noted the vitriolic nature of Bishop's remarks, see e.g., Dauben [51, p. 139], cf. footnotes 38, 75, and 43, M. Artigue [6, p. 172] described it as "virulent"; Davis and Hauser [56], as "hostile"; D. Tall [174, as "extreme".
} 
the usual definition of a limit is too complicated $\sqrt[33]{[26}$,

p. 208] [emphasis added-authors].

M. Davis notes that Bishop fails to acknowledge explicitly in his review in the Bulletin that his criticism is motivated by his foundational preoccupation with the law of excluded middle.34

A similar point is alluded to in Keisler's brief (and measured) response:

why did Paul Halmos, the Bulletin book review editor, choose a constructivist as the reviewer? [115, p. 269]

Keisler traced the source of Bishop's criticism, to the constructivist criticism of classical mathematics, more specifically of its reliance on classical logic, incorporating the key item rejected by intuitionists, namely the law of excluded middle. Comparing the use of the latter to wine, Keisler compares Halmos' choice of reviewer, to "choosing a teetotaller to sample wine". Halmos' reply will be analyzed in Subsection 3.2.

In short, the constructivist Bishop is criticizing apples for not being oranges: the critic and the criticized are not operating in a common foundational framework 35 The foundational framework of nonstandard analysis, namely the Zermelo-Fraenke 36 set theory with the axiom of choice (ZFC), is the framework of the vast majority of the readers of the Bulletin, at variance with Bishop's preferred intuitionistic framework. From the vantage point of the latter, Bishop's debasement

\footnotetext{
${ }^{33}$ Bishop is referring to the extension principle and the transfer principle of nonstandard analysis. See Subsection 3.5 .

${ }^{34} \mathrm{M}$. Davis writes as follows: "Keisler's book is an attempt to bring back the intuitively suggestive Leibnizian methods (but see footnote 7) that dominated the teaching of calculus until comparatively recently, and which have never been discarded in parts of applied mathematics. A reader of Errett Bishop's review of Keisler's book would hardly imagine that this is what Keisler was trying to do, since the review discusses neither Keisler's objectives nor the extent to which his book realizes them. Bishop[, meanwhile,] objects to Keisler's description of the real numbers as a convenient fiction (without informing his readers of the constructivist context in which this objection is presumably to be understood)" [55. p. 1008] [emphasis added-authors]. See further Subsection 3.5.

${ }^{35}$ A similar point was mentioned by M. Davis [55], see footnote 34 .

${ }^{36}$ It is interesting to note a criterion of success of a theory of infinitesimals as proposed by Adolf Abraham Fraenkel and, before him, by Felix Klein. In 1908, Klein formulated a criterion of what it would take for a theory of infinitesimals to be successful. Namely, one must be able to prove a mean value theorem for arbitrary intervals, including infinitesimal ones [119, p. 219]. In 1928, A. Fraenkel [70. pp. 116-117] formulated a similar requirement in terms of the mean value theorem. Such a Klein-Fraenkel criterion is satisfied by the Hewitt-Łoś-Robinson theory by the transfer principle, see Appendix A
} 
of meaning and obfuscation charges would apply equally well to most of classical mathematics, a point alluded to in Feferman's comment cited above 37 Perhaps one may be permitted to detect a deficiency in the attributes mentioned in the title of our current Section 3, when analyzing Bishop's published remarks concerning Robinson's approach.

3.2. Halmos's translation. An interesting exchange took place in the pages of the Bulletin and the Notices. Keisler's response to Bishop's review of his book was discussed in Subsection 3.1. Halmos' reply 38 to Keisler's question came in the form of an editorial pointer on p. 271 of the same issue, referring the reader to Halmos' outline of his editorial philosophy on p. 283:

As for judgments, the reviewer may [... s say (or imply) what he thinks.

Halmos's philosophy was that a reviewer may legitimately use the review of a book as a springboard for developing his own ideological agenda.

According to a close associate 63] of Halmos', Halmos' policy was to confront opposing philosophies in the goal of livening up the debate. One of his goals was to boost lagging sales that were plaguing the publisher at the time, see 89. The bottom-line issue, combined with Halmos' own unflattering opinion of non-standard analysis as "a special tool, too special" [89, p. 204], apparently made the choice of Halmos' student (Bishop) as the reviewer, attractive to the editor.

In the case of Keisler's book, such editorial philosophy translated into a review, by Bishop, whose thinly disguised foundational agenda (an attempt to de-legitimize the use of the law of excluded middle in mathematical practice) took the form of vitriolic criticism of Robinsonstyle infinitesimal calculus.

In his autobiography, Halmos described non-standard analysis as a special tool, too special [89, p. 204]. In fact, his anxiousness to evaluate Robinson's theory may have involved a conflict of interests. In the early 1960s, Bernstein and Robinson [19] developed a non-standard proof of an important case of the invariant subspace conjecture of Halmos'. In a race against time, Halmos produced a "standard translation" of the

\footnotetext{
${ }^{37}$ See footnote 31 for Feferman's comment.

${ }^{38}$ An additional reply to Keisler's query, by G. Stolzenberg [166] (a close associate of Bishop's), occupies slightly over a column on page 242 of the Notices. Given its author's interest 167] in literary deconstruction, we note that the reply manages to employ a root, dogma (conspicuously absent from Keisler's letter), on five occasions, culminating in the expression the spouting of dogma in the penultimate paragraph, cf. footnotes 32 and 75 .
} 
Bernstein-Robinson argument, in time for the translation [90] to appear in the same issue of Pacific Journal of Mathematics, alongside the original. Halmos invested considerable emotional energy (and "sweat", as he memorably puts it in his autobiography [89]) into his translation. Whether or not he was capable of subsequently maintaining enough of a detached distance in order to formulate an unbiased evaluation of non-standard analysis, his blunt unflattering comments may create an impression of impropriety, as if he were retroactively justifying his translationist attempt to deflect the impact of one of the first spectacular applications of Robinson's theory.

3.3. Why was non-standard analysis the target? We saw that Bishop's debasement charge applies to all of classical mathematics 39 What was it specifically about non-standard analysis, among other fields of classical mathematics, that may have prompted E. Bishop to speak out against it specifically? We argue in Subsection 3.5 that Bishop's comments on non-standard analysis in [25] and [26] need to be understood in the context of his foundational writings that appeared elsewhere, as neither his motivation, not his terminology, are explained in [25] and [26].

We will first mention three possible technical reasons for Bishop's choice of target, and then discuss a possible philosophical reason. The possible technical reasons as the following:

(1) Certain results of classical analysis can be convincingly adjusted to increase their numerical content, by strengthening their hypotheses and/or weakening the conclusions (cf. Subsection 4.1); meanwhile, Bishop apparently felt that such an option is unavailable for Robinson-style infinitesimals, leading to his wholesale rejection of the latter.

(2) A related point is that the hyperreal approach incorporates an element of non-constructivity at the basic level of the very number system itself.

(3) By replacing Weierstrassian epsilontics 40 by a theory of infinitesimals, non-standard analysis thereby removes the bread and butter of constructive analysis, starting with the constructive epsilontic definition of a Cauchy sequence, which involves an explicit rate of convergence, see [23].

\footnotetext{
${ }^{39}$ See also footnotes 45,52 , and 80 ,

${ }^{40} \mathrm{~A}$ term used by Dauben [51]. The description of "epsilon, delta" arguments as epsilontics (alternatively, epsilonics) is frequently found in the mathematical literature, and even in the names of courses of instruction at certain North American universities.
} 
Recall that Brouwer sought to incorporate a theory of the continuum as part of intuitionistic mathematics, by dint of his free choice sequences. Bishop's commitment to integr-ity (see glossary in Subsection 6.2) led to his dismissal of Brouwer's continuum as a "semimystical theory" 41 Such a commitment on Bishop's part could hardly have countenanced an about-face in the matter of the far denser thicket of Robinson's hyperreal continuum.

More fundamentally, we would like to argue that non-standard analysis presents a formidable philosophical challenge to constructivism, which may, in fact, have been anticipated by Bishop himself in his foundational speculations, as we explain below.

Bishop's vision of mathematics is at variance both with Kronecker's constructivist program (see Subsection 2.1) and with Brouwer's intuitionism. Thus, Bishop's constructive mathematics claims to be uniquely concerned with finite operations on the integers. Meanwhile, Bishop himself speculated that "the primacy of the integers is not absolute":

It is an empirical fact that all [finitely performable abstract calculations] reduce to operations with the integers. There is no reason mathematics should not concern itself with finitely performable abstract operations of other kinds, in the event that such are ever discovered [24, p. 53] [emphasis added-authors].

Bishop hereby acknowledges that the primacy of the integers is merely what he describes as an empirical fact. The implication is that the said primacy could be contradicted by novel mathematical developments. Perhaps Bishop sensed that a rigorous theory of infinitesimals is both

- not reducible to finite calculations on the integers, and yet

- accomodates a finite performance of abstract operations,

thereby satisfying his requirements for legitimate mathematics. Bishop made a foundational commitment to the primacy of the integers (a state of mind known as integr-ity, see Subsection 6.2) through his own work and that of his disciples starting in the late sixties. He may therefore have found it quite impossible, in the seventies, to acknowledge the existence of "finitely performable abstract operations of other kinds". We will analyze the related issue of post-LEM numerical meaning in Subsection 5.2 .

\footnotetext{
${ }^{41}$ See main text at footnote 60 for the full quote.
} 
3.4. Galley proof "debasement". It has recently come to light that Bishop's "debasement" remark, published in his Crisis text [25], in reality was never uttered in his oral presentation in the presence of the distinguished audience 42 at the 1974 workshop in Boston. A careful reading of Bishop's Crisis text already suggests that his "debasement" comment may have been added at the galley proof stage, as its deletion from the text causes no interruption of textual continuity. In fact, the second-named author has been able to locate a participant in the workshop, who confirmed that Bishop said nothing about nonstandard analysis in his oral presentation. The participant in question, a historian of mathematics, wrote as follows on the subject of Bishop's statement published in 1975 on Robinson-style infinitesimal calculus:

I do not remember that any such statement was made at the workshop and doubt seriously that it was in fact made. I would have pursued the issue vigorously, since I had a particular point of view about the introduction of non-standard analysis into calculus. I had been considering that question somewhat in my attempts to understand various standards of rigor in mathematics.

The statement would have fired me up [131.

Furthermore, the text [123], entitled Commentary on E. Bishop's talk, states clearly that the said commentary was based on a transcript of Bishop's actual talk at the workshop. When the second-named author contacted the authors of the said Commentary in the summer of 2009, it turned out that neither was aware of the fact that the published version of Bishop's Crisis essay contained a paragraph on the subject of non-standard analysis 43 Thus, Bishop appears to have added his

\footnotetext{
${ }^{42}$ See footnote 78

${ }^{43}$ In fact, Stolzenberg replied on 23 june 2009 to the effect that he is "morally certain [that Bishop] didn't mention non-standard analysis during that lecture". The second-named author subsequently requested a copy of the transcript of Bishop's lecture (on which the Commentary [123] was based). The reply arrived on 17 august 2009, and consisted of three parts:

(1) it acknowledged that the authors are in possession of such a transcript;

(2) it denied the request for a copy of the transcipt;

(3) it expressed concern about "the harm" that would result if this writer were to obtain a copy of the trascript.

The tenor of these remarks suggests that in fact the transcript of Bishop's talk contains no mention of non-standard analysis whatsoever, which would therefore prove that the addition of the vitriolic paragraph was an afterthought. The community of historians of mathematics should encourage Stolzenberg to release the transcript of Bishop's lecture in Stolzenberg's possession, as it represents an important historical document.
} 
"debasement" comment on non-standard calculus at the galley proof stage of publication.

To be sure, additional material is frequently added at galley stage. Was the addition of the "debasement" comment ethically problematic? Note the published version of Bishop's lecture includes an epilogue. The epilogue contains the discussion (following the lecture), involving Birkhoff, Mackey, J.P.Kahane, and others, who have challenged Bishop on a number of points. None of the participants challenged Bishop on his criticism of infinitesimals à la Robinson. This reader at least of Bishop's essay was disappointed, upon his first reading of the essay, not to find any challenge to Bishop's "debasement" of non-standard analysis, in the discussion that ensued. We now understand why there was no such challenge: Bishop did not say a word about Robinson in his oral presentation.

The important ethical point concerns the juxtaposition of Bishop's vitriolic comment (added at galley stage) concerning non-standard analysis, and an absence of any reaction to it in the ensuing discussion. Such a juxtaposition is misleading, as it suggests, if not agreement, then at least a tolerance toward Bishop's comment on the part of the participants in the discussion panel, raising issues of integrity (without the dash) 44 The vitriolic tenor of Bishop's remark was aimed ostensibly at Robinson-style infinitesimal calculus, but logically would apply equally well to the rest of mainstream mathematics, as we discuss in the next subsection 45

3.5. A hypothesis and its refutation. Bishop diagnosed classical mathematics with a case of a "debasement of meaning" in his Schizophrenia in contemporary mathematics, written in 197346 Hot on the heels of Schizophrenia came the 1975 Crisis in contemporary mathematics [25], where the same "debasement of meaning" diagnosis was slapped upon infinitesimal calculus à la Robinson. It is therefore transparent that his opposition to Robinson-style infinitesimals is merely an instance of a broad opposition to classical mathematics as a whole, on ideological/foundational grounds.

\footnotetext{
${ }^{44}$ Bishop should have been aware of the fact that the follow-up discussion will be included in the published version of his lecture. In order to avoid misunderstandings, he could have easily included a footnote to the effect that the non-standard paragraph was added at the galley proof stage, but he didn't.

${ }^{45}$ See Section 3.5 and Feferman's comment cited in footnote 31, as well as footnotes 39, 52, and 80.

${ }^{46}$ See Bishop [27, p. 1].
} 
Nonetheless, a hypothesis has been advanced by a number of constructivist 47 that Bishop's criticism of infinitesimal calculus à la Robinson was motivated primarily by pedagogical, rather than foundational, concerns. According to such a hypothesis, his objection focused on the use of the extension principle and the transfer principle of nonstandard analysis (see Appendix (A), whose acceptance by the student would necessarily be on the grounds of trust in the teacher 48

However, such a hypothesis does not stand up to scrutiny, as we argue below. A widespread perception of a foundational motivation behind Bishop's criticism of non-standard analysis has been attested to by M. Davis, V. Komkov, J. Dauben, H. J. Keisler, D. Tall, and others, and is not a novel interpretation.

Thus, M. Davis comments that Bishop states his objections "without informing his readers of the constructivist context in which this objection is presumably to be understood" [55, p. 1008]. Physicist V. Komkov notes that

Bishop is one of the foremost researchers favoring the constructive approach to mathematical analysis. It is hard for a constructivist to be sympathetic to theories replacing the real numbers by hyperreals [122, p. 270] 49

Philosopher of mathematics G. Hellman writes:

Some of Bishop's remarks (1967) suggest that his position belongs in [the radical constructivist] category [95, p. 222].

Historian of mathematics J. Dauben analyzed Bishop's criticism and found it to be "unfounded" 50

\footnotetext{
${ }^{47}$ Including Stolzenberg [166].

${ }^{48}$ It has been argued further that the idea of constructive mathematics is to found mathematics on actions that everyone can concretely perform, in such a way that mathematics is accessible for everyone. Let us examine the coherence of such an argument (certain implications of a Bishop-style constructive approach in teaching is examined in Subsection 4.1). The assumption of a completed (actual) infinity of $\mathbb{Z}$ in Bishopian constructivism is a departure from Kronecker's vision. It cannot be "performed" in any concrete sense by either student or teacher. Nevertheless, both constructivists and classical mathematicians acknowledge that such an assumption facilitates both teaching and research in mathematics.

${ }^{49}$ Leaving aside the issue whether or not non-standard analysis can be done constructively, Komkov's perception of a foundational concern on Bishop's part is unmistakable.

${ }^{50}$ After evoking the success of nonstandard analysis "at the most elementary level at which it could be introduced-namely, at which calculus is taught for the first time", Dauben proceeds to point out that "there is also a deeper level of meaning at which nonstandard analysis operates." Dauben mentions the impressive
} 
Through a comparative textual study of Bishop's three texts, we show that his criticism of non-standard analysis was motivated more by foundational than by pedagogical issues.

1. Bishop's perception of a shared shortcoming in non-standard calculus, on the one hand, and classical mathematics pedagogy, on the other, is evident from his contention that the former will

confirm the students' previous experience [26, p. 208]

[emphasis added-authors].

of the latter. The usage of the term confirm points to a shared shortcoming.

2. The nature of such a shared shortcoming is hinted upon in his reference to the students' experience with classical mathematics as a "meaningless exercise in technique" [26, p. 208] [emphasis addedauthors].

3. Note that the term meaning is alluded to elsewhere in his review, as well. Thus, he laments that in "our educational system", it is "a bad form to ask what it all means" [26, p. 206]. The 1977 review is evasive as to what exactly Bishop has in mind when he mentions meaning.

4. Meanwhile, Bishop's Crisis essay [25] from 1975 focuses on the virtue of numerical meaning, defined ultimately as the rejection of the law of excluded middle even in its special case called LPO 51 Bishop criticized non-standard calculus as a "debasement of meaning" in the following terms:

It is difficult to believe that debasement of meaning could be carried so far [25, 514] [emphasis addedauthors].

Bishop's wording suggests that debasement of meaning had already been spoken of, while, allegedly, this particular instance of it, is carried particularly far. However, the Crisis text does not contain any other occurrences of the three-word formation. Where was debasement of meaning spoken of previously by Bishop?

5. The realm of applicability of the three-word formation is clarified in Bishop's essay Schizophrenia in contemporary mathematics [27]. The

applications in "physics, especially quantum theory and thermodynamics, and in economics, where study of exchange economies has been particularly amenable to nonstandard interpretation" (some references for such applications can be found in Appendix A . At this deeper level of meaning, Dauben concludes: "Bishop's views can be questioned and shown to be as unfounded as his objections to nonstandard analysis pedagogically" [47, p. 192].

${ }^{51}$ See footnote 31 for a more detailed discussion of Bishop's LPO. 
essay was published posthumously in 1985, but M. Rosenblatt [154, p. ix] points out that it was "distributed" in 1973. In other words, Bishop's Schizophrenia text was distributed a year or two prior to the Crisis text. Bishop writes as follows:

Brouwer's criticisms of classical mathematics were concerned with what I shall refer to as "the debasement of meaning" [27, p. 1].52

In Bishop's own words, the debasement of meaning expression, employed in his Crisis text to refer to non-standard calculus, is in fact a criticism of classical mathematics as a whole 53 The three-word formation sailed effortlessly from Schizophrenia to Crisis, revealing its author's intention.

6. By the time Bishop got around to describing non-standard calculus as a debasement of meaning [25, p. 514], he had already used the term meaning as shorthand for numerical meaning in his invocation of a philosophical principle, to the effect that a discussion of meaning should precede a discussion of truth [25, p. 509].

7. Bishop's remark about meaning preceding truth occurs in the context of his analysis of the Brouwer-Hilbert controversy, which he attributes to the fact that

"[t]hey attached different meanings to Cantorian set theory" [25, p. 509] [emphasis added-authors]

8. Bishop provides an example explaining the meaning of an integer to Brouwer:

"[f]or example, an integer to Brouwer [...] is either a[ concrete] integer in decimal notation or a method that in principle will lead after a finite number of steps to an integer in decimal notation. Again there is this notion of computability: if the integer is not given directly to be sure that it is finitely computable." [25, p. 509-510] [emphasis added-authors]

\footnotetext{
${ }^{52}$ See also footnotes 39,45 , and 80.

${ }^{53}$ When Bishop's usage of the identical expression, debasement of meaning, in the context of both non-standard calculus and classical mathematics, was presented in 2009 (as evidence of the claim of a foundational motivation on Bishop's part in criticizing non-standard calculus) to a constructivist and a close associate of Bishop's, he responded as follows: "Classical math here; non math major calculus there. Two different kinds of debasement of meaning". See footnote 75.
} 
9. Toward the end of an imaginary dialog between Brouwer and Hilbert, Bishop has Brouwer express himself as follows on the subject of meaning:

For me to do my mathematics in your system would entail a significant loss of meaning 54 [25, p. 511].

To Brouwer, as interpreted by Bishop, meaning is seen through the lens of notions of computability, numerical meaning, and LEM. Meanwhile, Hilbert is uninterested in LEM extirpation; for him, the source of meaning lies elsewhere 55

10. Thus, the shared shortcoming of infinitesimal calculus à la Robinson and classical mathematics, in Bishop's eyes, ultimately boils down to a deficiency in numerical meaning, in other words, to a reliance on LEM.

Through a comparative textual analysis of his three texts, we have argued that Bishop's grievance is foundationally motivated, even when couched in a pedagogical idiom. Indeed, foundational ideology and pedagogy were inseparable in Bishop's thinking.

Our conclusion is that the Bishop-Keisler controversy was, in essence, a foundational controversy, as suggested by J. Dauben, see [47, p. 177],

\footnotetext{
${ }^{54}$ Bishop does not speculate what Hilbert's reply might have been to such a charge of a loss of meaning, but we cannot help noting that for a classical mathematician to do mathematics in Brouwer's system, would entail a significant loss of post-LEM numerical meaning. Thus, H. Weyl, who became disillusioned with the Brouwerian development by the 1930s, notes that "Mathematics with Brouwer gains its highest intuitive clarity. He succeeds in developing the beginnings of analysis in a natural manner, all the time preserving the contact with intuition much more closely than had been done before. It cannot be denied, however, that in advancing to higher and more general theories the inapplicability of the simple laws of classical logic eventually results in an almost unbearable awkwardness. And the mathematician watches with pain the larger part of his towering edifice which he believed to be built of concrete blocks dissolve into mist before his eyes" [190, p. 54] (see further in Feferman [67]). See also Subsection [5.2, and particularly Example 5.3

${ }^{55}$ Thus, Novikov [138] writes that, for all of Hilbert's interest in a program of formalisation and axiomatisation, he understood such a program in a highly nontrivial fashion. Novikov mentions, notably, Hilbert's deep theorem 103 to the effect that the Einstein equations of relativistic gravitation are of Lagrangian type. Novikov's essay is discussed further in Section 8.2. A detailed discussion of the significance, and meaning, of Hilbert's program may be found in Avigad and Reck [10. See also Corry's quote of Hilbert (footnote 27).
} 
[47, p. 190], and [47, p. 192],56 as well as by V. Komkov The controversy involved a fundamental disagreement concerning a basic question, which can be formulated in terms of the dichotomy introduced in Subsection 5.2, as follows. Is post-LEM numerical meaning, mathematically coherent?

In conclusion, Bishop's vitriolic criticism of infinitesimals à la Robinson was but a salvo in his broadside attack on classical analysis and classical mathematics as a whole, percolating through a number of his foundational texts, and culminating in the posthumously published charge of Schizophrenia [27].

3.6. Further background on refuted hypothesis. In the previous section, we argued that Bishop's criticism [25], [26] of non-standard calculus was foundationally motivated. Here we provide some background on the context of Bishop's review, and clarify some of the pedagogical and mathematical issues involved.

Bishop's Crisis 25] tells an epic tale of how, half a century earlier, a failure of communication between the two giants (Brouwer and Hilbert), had, in Bishop's view, the effect of derailing mathematics from its numerical foundations, and set it on its present "meaningless" course. Crisis also presents a vision of how mathematics may have been rescued, had the Brouwer-Hilbert debate only followed a different script (namely, the one specified in [25]), focusing on the foundational issue of "principles of omniscience" and the problem of its acceptability.

In the midst of the epic tale, Bishop inserts 58 a vitriolic paragraph against Robinson-style infinitesimal calculus. To believe Bishop's followers, his unique concern is the best way to teach freshman calculus. While a certain suspension of disbelief is required here, it should also be pointed out that if the source of Bishop's interest were indeed pedagogical, then he pursued it with extraordinary nonchalance.

The remarks he added to the galley proofs 58 of [25] indicate that he was already aware of infinitesimals in the classroom, at least two years prior to writing his 1977 book review [26]. Meanwhile, K. Sullivan's 1976 field study [169] of infinitesimals in the classroom was published in a mainstream periodical, was easily accessible, and showed the infinitesimal approach to be more successful than the standard one in imparting an ability to interpret the sense of mathematical formalism of calculus. J. Dauben pointed out that

\footnotetext{
${ }^{56}$ See footnote 50 .

${ }^{57}$ See footnote 49 .

${ }^{58}$ See Subsection 3.4 .
} 
[Sullivan's] study [...] was presumably available to Bishop when his review of Keisler's book appeared in 1977, in which he attacked the pedagogical validity of nonstandard analysis [47, p. 190].

Dauben analyzes Sullivan's comparison of the nonstandard approach and the traditional approach, with the conclusion that

contrary to Bishop's views, the traditional [epsilontic] approach to calculus may be the more pernicious [47, p. 191].

Why wasn't Sullivan's study dealt with, one way or another, in Bishop's book review? Could it be because Bishop was not interested in field studies? Could his opposition to the non-standard approach have been based, not on any of its perceived shortcomings in imparting knowledge of rates of change, derivatives, areas, integrals, etc., but rather on ideological and foundational grounds? That a certain amount of foundational material should be expected to be taken on trust in a first calculus course, is a feature that infinitesimal-based calculus shares with the standard approach. Very rare indeed is the first calculus course that deals with equivalence classes of Cauchy sequences (or other constructions of the real number field). In the decade of his life that Bishop seems to have become interested in calculus methodologies, he is not known to have written critical book reviews of any methodologies other than the hyperreal one, which-curious coincidence!-incorporates an element of non-constructivity at the basic level of the very number system itself 59

Having already written, about Bertus Brouwer himself, that

"Brouwer's bugaboo has been compulsive speculation about the nature of the continuum. His fear seems to have been that, unless he personally intervened to prevent it, the continuum would turn out to be discrete. [The result was Brouwer's] semimystical theory of the continuum" 60 [23, p. 6 and 10],

was Bishop likely to take lying down, a far denser thicket of Robinson's hyperreals? Could his interest have been foundationally motivated, as indeed it was perceived by many of the readers of the Bulletin, as is readily acknowledged even by Bishop's followers?

To elaborate, note that Bishop quotes Keisler's observation that

\footnotetext{
${ }^{59}$ See footnote 63 for Robinson's take on number systems.

${ }^{60}$ See also main text at footnote 41
} 
"we have no way of knowing what a line in physical space is really like. It might be like the hyperreal line, the real line, or neither. However, in applications of the calculus, it is helpful to imagine a line in physical space as a hyperreal line."

Bishop himself does not comment, other than voicing a criticism as to an alleged lack of supporting evidence. His presentation suggests that Bishop himself favors the classical real line. Yet Bishop states clearly in his Manifesto [23] that from his constructivist viewpoint, it is LEM, and not the axiom of choice, that is the source of non-constructivity 61

To summarize, Bishop seeks to convince his classical reader of the idealistic nature (see glossary in Section 6.2) of a Robinson infinitesimal, whereas to Bishop himself, the classical continuum 62 should not "have been put there in the first place" any more than a Robinson infinitesimal, as the classical construction of both relies on the law of excluded middle. The similar foundational status of the classical continuum, on the one hand, and the hyperreal number, on the other, constitutes an astonishing point of convergence between Bishop and Robinson 63

On the subject of Keisler's infinitesimals, Bishop had this to say:

We are not told what an infinitesimal $\Delta x$ is [...] Keisler has developed limits from a supposedly consistent system of axioms [...] But he has not explained the axioms. They are mere conveniences for generating proofs, whose intuitive content will certainly escape the students [26. p. 207] [emphasis added-authors].

What exactly is the source of Bishop's certainty? The point is not so much that, having taught calculus effectively, first-hand, from Keisler's book, this author disagrees with Bishop's assessment as to the effectiveness of Keisler's method 64 Rather, the point is that the nature, and the language, of Bishop's criticisms clearly reveal an axiomatic/foundational preoccupation.

\footnotetext{
${ }^{61}$ See our main text around footnote 70 .

${ }^{62} \mathrm{He}$ felt similarly about Brouwer's continuum; see footnote 24 and the main text at footnote 60.

${ }^{63}$ Concerning the reality of number systems, Robinson expressed himself as follows: "[T]he infinitely small and infinitely large numbers of a non-standard model of Analysis are neither more nor less real than, for example, the standard irrational numbers $[\ldots]$ both standard irrational numbers and non-standard numbers are introduced by certain infinitary processes" [148, p. 282].

${ }^{64}$ See [109, 110] for details.
} 
Dauben [51] also understood Bishop's "debasement of meaning" criticism of non-standard calculus as referring to an alleged lack of numerical meaning 65 The latter is Bishop's term for the sine qua non ingredient of constructively acceptable mathematics. The lack of numerical meaning, to Bishop, corresponds to a reliance on LPO or more generally, the law of excluded middle (see glossary in Subsection 6.2).

The over-reliance on the teacher's authority, in Bishop's view, is only a shortcoming if such authority is propped up by a non-constructive arsenal such as excluded middle or classical choice. Bishop expresses the sentiment that

the notorious $\epsilon, \delta$ definition of limit is common sense

[26, p. 208] [emphasis added-authors].

He further shares with the reader the following confidence: "[the students] don't believe me". Dauben speculates that there may be a very good reason for their disbelief, namely,

that what [Bishop] claims, in fact, does not seem to be true [51, p. 134].

The vast education literature on the student reception of quantifiers (see a useful bibliography in [187]) attests to the irreducible difficulty of properly inculcating an understanding of even a single alternation of quantifiers, let alone an iteration of such alternations involved in the $\epsilon, \delta$ definition 66

However, our main goal here is not to gauge the difficulty of Weierstrassian definition of the limit concept based on epsilontics. Rather, the point is to signal the nonchalance of Bishop's comments, if they are to be interpreted as pedagogically motivated.

Interpreted as foundationally motivated, on the other hand, Bishop's comments become intelligible. In traditional (LEM-dominated) mathematics courses, students become accustomed to going through the numerically meaningless motions of idealistic mathematics (see glossary in Section 6.2), so that by the time they reach Bishop's classroom and are exposed to the numerically meaningful content of Weierstrassian epsilontics, they are put off by its pristine "common sense". As part of a constructivist critique of the foundations, Bishop's criticism is intelligible and compelling, even if ultimately flawed, as we argue in Section 8 ,

For all its touted advantages of increasing numerical meaning by means of a constructivisation of all that can be constructivized, Bishop's

\footnotetext{
${ }^{65}$ See footnote 50 .

${ }^{66} \mathrm{~A}$ detailed discussion of a typical case study appears in the main text at footnote 69 .
} 
approach is not without its pitfalls. Note that a "de-basement" of mainstream mathematics is what constructivism is sometimes attempting to carry out, by changing the meaning of basic terms such as continuous function and finite set, as analyzed in Sections 4.1 and 4.2 .

\section{De-BAsing in CONSTRUCTIVISM}

A number of familiar terms in the mathematical lexicon are displaced from their base, or basic, meaning when they are rendered constructive. We will analyze several such phenomena in this section.

4.1. Verbal equality. Bishop's own approach to constructive mathematics only accepts uniformly continuous functions (more precisely, uniformly continuous on compact intervals); they are accordingly renamed continuous in Bishop's foundational text [23]67 S. Feferman writes that Bishop deals with

a very special class of functions of real numbers, namely those which are uniformly continuous on [e]very compact interval. In this way, he finesses the whole issue of how one arrives at Brouwer's theorem [to the effect that every function on a closed interval is uniformly continuous 68 by saying that those are the only functions, at least initially, that one is going to talk about [66].

Continuity differs from uniform continuity in at least the following three ways:

(1) continuity can be defined in terms of limits;

(2) continuity is a pointwise concept;

(3) only 3 quantifiers are required to define continuity at a point.

To elaborate, note that a typical calculus textbook [180, p. 108] defines continuity in terms of a previously defined notion of the limit. This is possible because ordinary continuity is a local (pointwise) property, whereas to define uniform continuity, one needs to work with pairs of points. Thus, the definition of uniform continuity [180, p. 115] requires a return to $\epsilon, \delta$ 's.

Most textbooks adopt the standard Weierstrassian approach using epsilontics. When it comes to continuity and uniform continuity, both

\footnotetext{
${ }^{67}$ While Bishop, at the time of writing [23, appears to have thought that such functions were the only ones that would enter into numerically meaningful mathematics, subsequent research has shown that even sequential continuity (constructively strictly weaker than even pointwise continuity) arises naturally in constructive functional analysis; see Ishihara [106].

${ }^{68} \mathrm{~A}$ discussion of the issue of the finessing of Brouwerian counterexamples in the context of the extreme value theorem appears in Subsection 4.3
} 
definitions involve alternations of quantifiers. However, pedagogically speaking, one can present the definition of ordinary continuity with only three quantifiers, once the point has been fixed. There is no such device that would permit a definition of uniform continuity with fewer than four quantifiers. Such a definition is almost inaccessible for an average undergraduate.

A revealing case study [69] documents an effort properly to explain $\epsilon, \delta$ to students at "a state university with an enrollment of 15,000 ", using an interactive approach that reportedly generated considerable interest on the part of the students. It emerges that due to the intrinsic difficulty of the subject matter, the semester was spent explaining $\epsilon, \delta$ for linear functions. The course itself never reached quadratic functions, but a number of students "began coming to office hours on a regular basis" to get a taste of the quadratic case. The author concludes that

[a]lthough the ideas concerning quadratics were pursued outside of class with only a limited number of students, it is important to note that the students initiated this discussion and pursued it on their own time [69, p. 53] 69

For educators, it is no mystery why Johnny can't take limits (see Roh [153]). Many teachers, based on classroom experience, feel that uniform continuity is a concept that is even more difficult for students to grasp than ordinary continuity.

Bishop's renaming of uniformly continuous functions as continuous, is part of a pattern commented upon by reviewer B. van Rootselaar (for Mathscinet) of Bishop's book [23], who writes as follows:

The comparison with classical mathematics carried out by the author in the notes accompanying the chapters is somewhat superficial. In fact the author stresses the equal hypothesis interpretations of classical theorems, which is misleading since the hypotheses are for the greater part only verbally equal [182].

What van Rootselaar is referring to is the fact that some of the theorems are worded in such a way as to sound identical to the classical ones, but, of course, the definitions have been tampered with, e.g. what is defined as continuity is really uniform continuity, classically speaking (See also Hellman [93]).

\footnotetext{
${ }^{69}$ There are indeed numerous case studies of this sort; see e.g. [187.
} 
Apart from the issue of verbal equality, the numerical advantage of Bishop's approach has been neatly summarized by Feferman, who pointed out that in constructive analysis,

concepts are chosen so that there is a lot of witnessing information introduced in a way that is not customary in classical mathematics, where it is hidden, for instance, by implicit use of the axiom of choice [66].

4.2. Goodman-Myhill's $1 \frac{1}{2} \pm \frac{1}{2}$ sets and "full" choice. What does the term finite mean? In this section we will comment on the constructive distinction between finite and subfinite sets, in the context of a result relating the axiom of choice and LEM.

A theorem dating from 1975, alternatively referred to as the the Diaconescu theorem [57] or the Goodman-Myhill theorem [81, asserts that the axiom of choice implies the law of excluded middle.

There is an intriguing point here that Bishop actually claimed that he has no quarrel with the axiom of choice. In his Constructive manifesto, eight years prior to the publication of Diaconescu-Goodman-Myhill, he wrote:

the axiom of choice $[\ldots]$ is not a real source of the unconstructivities of classical mathematics [...] [it] is used to extract elements from equivalence classes where they should never have been put in the first place [23, p. 9].

His remark has apparently been a source of discomfort for some of his followers 70 Understanding the proof of the Diaconescu-GoodmanMyhill theorem hinges on the following critical distinction between finite and subfinite sets 71 A set possessing either one or two elements (we don't know which) cannot be called finite in the constructive sense. Finding a choice function for a collection of sets containing either one or two sets (we don't know which) requires a nontrivial application of the "full" axiom of choice. The theorem is proved by means of such an application of the axiom of choice (see Subsection 2.3 for a discussion

\footnotetext{
${ }^{70}$ It appears to some, if not all, constructive interpreters that Bishop's remark was, if studied carefully, perfectly in order, if less than perfectly expressed; see 28 , p. 13].

${ }^{71}$ The constructivist position on the issue can be summarized as follows. The contention that the image of a finite set under a function $f$ need not be finite, has precise meaning in a constructive context, reflecting a deficiency of numerical meaning in a classical assertion of its finiteness.
} 
of the nature of the quantifiers in the axiom of choice) 72 Note that we are dealing with a collection of sets containing $1 \frac{1}{2} \pm \frac{1}{2}$ sets (namely, either 1 or 2 sets) 73

Note that the law of excluded middle is a constructive consequence of much more elementary statements than that of the full axiom of choice.74

\subsection{Is Bishopian constructivism compatible with classical logic?}

We will examine the question of such compatibility in this subsection, in the context of the extreme value theorem. Note that the extreme value theorem does not hold constructively. Namely, the existence of a maximum of a continuous function $f$ on $[0,1]$, in the sense of the constructive formula

$$
\left(\exists_{\mathrm{InLo}} x \in[0,1]\right)(f(x)=\sup (f)),
$$

does not hold. Indeed, let $a$ be any real number such that

$$
a \leq 0 \text { or } a \geq 0 \text { is unknown. }
$$

Next, define $f$ on $[0,1]$ by

$$
f(x):=a x
$$

Then $\sup (f)$ is simply $\max (0, a)$, but the point where it is attained cannot be captured constructively (see [181, p. 295]). To elaborate on the foundational status of this example, note that the law of excluded middle: $P \vee \neg P$ ("either $P$ or (not $P$ )"), is the strongest principle rejected by constructivists. A weaker principle is the LPO (limited principle of omniscience). The LPO is the main target of Bishop's criticism in [25]. The LPO is formulated in terms of sequences, as the principle that it is possible to search "a sequence of integers to see

\footnotetext{
${ }^{72}$ For the benefit of a classically-trained reader it may be helpful to clarify that the "fullness" of the power of the axiom of choice referred to here is not in the usual sense, of applying it to a collection of sets of arbitrarily high cardinality, but rather to the omniscience aspect of the axiom of choice (see discussion of LPO in Subsection 3.1), stemming from the classical interpretation the the existence quantifier.

${ }^{73}$ The distinctions made by constructive mathematicians are real and important if you want to work constructively. Yet they often look odd if you cannot step outside the classical framework.

${ }^{74}$ Let $P$ be any statement, and let $S=\{0\} \cup\{x: x=1 \wedge(P \vee \neg P)\}$. Then $S$ is a subset of $\{0,1\}$ that certainly contains 0 ; but $1 \in S$ if and only if $P \vee \neg P$ holds. Thus if under the Brouwer-Heyting-Kolmogorov interpretation of logic, we could prove that every inhabited subset of $\{0,1\}$ either contains exactly one element, or contains both 0 and 1 , then we could prove the law of excluded middle. So the law of excluded middle is a constructive consequence of much more elementary statements than that of the full axiom of choice.
} 
whether they all vanish" [25, p. 511]. The LPO is equivalent to the law of trichotomy:

$$
(a<0) \vee(a=0) \vee(a>0)
$$

An even weaker principle is

$$
(a \leq 0) \vee(a \geq 0),
$$

whose failure is exploited in the construction of the counterexample under discussion. This property is false intuitionistically. After discussing real numbers $x \geq 0$ such that it is "not" true that $x>0$ or $x=0$, Bishop writes:

In much the same way we can construct a real number $x$ such that it is not true that $x \geq 0$ or $x \leq 0$ [23, p. 26], [28, p. 28].

The fact that an $a$ satisfying $\neg((a \leq 0) \vee(a \geq 0))$ yields a counterexample $f(x)=a x$ to the extreme value theorem (EVT) on $[0,1]$ is alluded to by Bishop in [23, p. 59, exercise 9]; [28, p. 62, exercise 11]. Meanwhile, Bridges interprets Bishop's italicized "not" as referring to a Brouwerian counterexample, and asserts that trichotomy as well as the principle $(a \leq 0) \vee(a \geq 0)$ are independent of Bishopian constructivism. See D. Bridges [33] for details; a useful summary may be found in Taylor [177].

The extreme value theorem illustrates well the fact that a verificational interpretation of the quantifiers in Bishopian mathematics necessarily results in a clash with classical mathematics; it is merely a matter of tactical emphasis that the clash is minimized in Bishopian constructivism.

\section{Are there tWO CONSTRUCTIVISMS?}

\subsection{Numerical constructivism or anti-LEM constructivism?}

G. Hellman has distinguished between liberal and radical versions of constructivism in [95]. The dichotomy 75 was picked up both by by H. Billinge [21] and by E. Davies [53]. Rather than borrowing terms from political science, we will exploit terms that are somewhat more self-explanatory in a mathematical context.

What are the goals of constructive mathematics? Bishop in his 1975 Crisis essay [25] emphasizes a quest for numerical meaning (see Subsection 3.1).

\footnotetext{
${ }^{75}$ While such a dichotomy seems to have taken root among the philosophers of mathematics, it may take time to penetrate the radical defenses.
} 
It seems reasonable to assume that a mainstream mathematician can relate favorably to the methodological approach that seeks greater numerical meaning, as enunciated by Bishop in [25]. Such an approach could be described as numerical constructivism, or rather, numerically meaningful constructivism. A methodological approach, complementing other approaches, could be a companion to classical mathematics, while at the same time recognizing the coherence of post-LEM numerical meaning (see Subsection 5.2).

On the other hand, when the emphasis shifts to the extirpation of the law of excluded middle from the mathematical toolkit, whether there is numerical benefit or loss 76 in such a quest, one arrives at an approach that could be termed anti-LEM constructivism, conceived of as an alternative to classical mathematics, and viewing LEM as a variety of mathematical phlogiston 77

To summarize, Bishop's comments could lead to two distinct conceptions of constructivism:

(1) numerical constructivism, a companion to classical mathematics; and

(2) anti-LEM constructivism, an alternative to classical mathematics.

Returning to Bishop's Crisis essay [25], we note that when he found himself in front of a distinguished audience at the Boston workshop 78 he chose to emphasize the goal of seeking greater numerical meaning over the goal of the elimination of LEM. Such an attitude is apparently consistent with Heyting's approach discussed above.

Elsewhere, however, Bishop adopts a different approach, implying that unless one extirpates LEM, one's mathematics falls in the range between obfuscation and debasement of meaning. In short, constructive mathematics is posited, not as a companion, but rather as a replacement for and alternative to, classical mathematics.

Should the gentle reader feel that our description of an anti-LEM species of constructivism is exaggerated, she should ponder H. Billinge's comment reproduced below. Billinge's sympathies for constructivism have led her to issue a philosophical challenge, so as

\footnotetext{
${ }^{76}$ Specifically, one loses the intuitively appealing infinitesimal definitions of the calculus, certain results of mathematical physics, the calculus of variations, soap films and bubbles (see Subsection [8.2), and other types of post-LEM numerical meaning discussed in Subsection 5.2 .

${ }^{77}$ See Subsection 6.1 on Pourciau's phlogiston metaphor.

${ }^{78}$ His audience included Birkhoff, Dieudonné, Freudental, J.P.Kahane, Kline, and Mackey.
} 
to settle the matter of whether we should permit or prohibit the use of classical mathematict 79 [20, p. 317] [emphasis added-authors].

Elsewhere, Billinge has claimed that E. Bishop is not a radical constructivist:

We can safely say that Bishop was a liberal constructivist [21, p. 187].

Yet, one wonders what Billinge would make of Bishop's published opinion to the effect that

Very possibly classical mathematics will cease to exist as an independent discipline [24, p. 54] 80

Furthermore, Bishop's vitriolic book review [26] is absent from Billinge's bibliography, and she makes no attempt to explain Bishop's "debasement" comments [25, p. 513-514] and [27, p. 1] targeting classical mathematics as a whole.

5.2. Hilbert and post-LEM meaning. To elaborate further on the difference between the two approaches to constructivism discussed in Subsection 5.1, we will examine the attitude toward calculus, from the perspective of the formalist/intuitionist debate. The infinitesimal definition of the derivative was already envisioned by the founders of the calculus, and was justified by A. Robinson (see Appendix A). Heyting is able to appreciate such an accomplishment (see Subsection 7.3), firmly grounded, as it was, in classical logic, making it unacceptable to an anti-LEM constructivist.

An infinitesimal definition certainly leads to computationally meaningful formulas for the derivatives of the standard functions, even though classical logic was relied upon by Robinson. To emphasize the difference between the two types of numerical meaning, we introduce the following distinction.

We will refer to the content of mathematics that is fully constructive from the bottom (i.e. integers) up, as pre-LEM numerical meaning, and to the content of mathematics that has indisputable numerical or

\footnotetext{
${ }^{79}$ Note that in Hellman's view [98, p. 439], "any [...] attempt to reinstate a 'first philosophical' theory of meaning prior to all science is doomed". What this appears to mean is that, while there can certainly be a philosophical notion of meaning before science, any attempt to prescribe standards of meaning prior to the actual practice of science, is doomed. See also discussion of Dummett in main text at footnote 105 .

${ }^{80}$ See also footnotes 39,45 , and 52 .
} 
computational meaning, while at the same time relying on LEM at some intermediate stage, as post-LEM numerical meaning 81

Pre-LEM numerical meaning is, of course, exemplified by Foundations of constructive analysis [23]. We will now present several examples of post-LEM numerical meaning, following Euclid, Leibniz, Hilbert, Loewner, Connes, Yau, and Avigad.

Example 5.1. An early example of what we consider post-LEM numerical meaning is given by geometric constructions in antiquity.

The volume calculations in Archimedes appear to rely on proofs by contradiction. Thus, a contradiction is derived from the assumption that the volume $x$ of the unit sphere is smaller than two-thirds of the volume of the circumscribed cylinder, and similarly for greater than. The contradiction proves that the volume is exactly two-thirds. The proof appears to depend on the trichotomy for real numbers:

$$
(x<y) \vee(x=y) \vee(x>y),
$$

where $y$ is two-thirds of the volume of the cylinder. We start with the trichotomy, eliminate the possibilities on the right and on the left, to conclude that the possibility in the middle (equality) is the correct one.

However, the proof can be modified slightly to meet the requirements of Bishop's framework. Thus, proving first that $\neg(x<y)$ implies $x \geq y$ (but not vice versa $)^{82}$ and $\neg(x>y$ ) implies $x \leq y$, to conclude that $x=$ $y$ without appealing to trichotomy.

Meanwhile, Euclid's constructions were recently examined, and found to be lacking, from a constructivist viewpoint, by M. Beeson [14. Beeson succeeds in reformulating much of Euclid in a constructively acceptable fashion, namely without "test-for-equality" constructions (such constructions rely on LEM so as to be able to consider the two cases $A=B$ and $A \neq B$ separately).

Beeson's work is no doubt a significant accomplishment. Yet one can question the ideological assumption that, due to their reliance on LEM, Euclid's arguments were somehow lacking in meaning prior to Beeson's arrival on the scene. Will such an assumption be shared by all of Beeson's readers?

\footnotetext{
${ }^{81}$ See main text preceding footnote 114 for an analysis of Richman's views in the light of our pre/post-LEM dichotomy.

${ }^{82}$ One cannot derive, constructively, $x<y$ by getting a contradiction from the assumption that $x \geq y$. Note that the formula $\neg(x<y) \rightarrow(x \geq y)$ implies its contrapositive $\neg(x \geq y) \rightarrow \neg \neg(x<y)$, but double negation $\neg \neg(x<y)$ does not imply $x<y$ if one is working constructively.
} 
Example 5.2. Leibniz's calculus was based on an apparently computationally questionable entity. Indeed, the theoretical entity called the infinitesimal appeared to have no empirical or computational counterpart 83 Thus, the algorithms for computing derivatives as developed by Leibniz, appear to rely upon non-constructive foundational material. Infinitesimal calculus remains in the post-LEM category even after Robinson's work, due to the nature of the construction of the hyperreal number system (but see Palmgren [139]).

Example 5.3. The Einstein-Hilbert action (alternatively, EinsteinHilbert Lagrangian) is the action that yields Einstein's field equations for the spacetime metric in general relativity. S. P. Novikov [138] writes that, for all of Hilbert's interest in a program of formalisation and axiomatisation, he understood such a program in a highly nontrivial fashion. Novikov mentions, notably, Hilbert's deep theorem [103] to the effect that the Einstein equations of relativistic gravitation are of Lagrangian type. The equations are thereby placed in the context of a variational problem. Novikov's essay is discussed further in Section 8.2, in the context of the general difficulty of accounting constructively for variational principles 84 This is only the most famous example of a meaningful variational problem that possesses an undeniable meaning of a post-LEM variety.

Example 5.4. Loewner's torus inequality (see Katz [108]) is proved using the uniformisation theorem, which is not fully constructive. Yet the inequality itself possesses indisputable numerical meaning. The inequality relates a pair of metric invariants of a Riemannian 2-torus $\mathbb{T}$. The first is the least length, denoted Sys, of a loop on $\mathbb{T}$ that cannot be contracted to a point on $\mathbb{T}$. The second invariant is the total area, denoted Area, of $\mathbb{T}$. The inequality, Sys $^{2} \leq \frac{2}{\sqrt{3}}$ Area, is illustrated in Figure 8.1. Mikhail Gromov writes as follows:

I was exposed to metric inequalities in the late 60's by $\mathrm{Yu}$. Burago, who acquainted me with the results of Loewner, $\mathrm{Pu}$, and Besicovitch. These attracted me by the topological purity of their underlying assumptions,

\footnotetext{
${ }^{83}$ This aspect of the infinitesimal was a source of Berkeley's metaphysical criticism (Berkeley's criticism was dissected into its metaphysical and logical components by D. Sherry 161]). Berkeley's empiricist philosophy stemming from his perception-based theory of knowledge, tolerated no theoretical entities without an empirical referent.

${ }^{84}$ With hindsight, it can be said that a critique of intuitionism based on variational principles, rather than boxer's fists, may have been a more effective critique by Hilbert.
} 
and I was naturally tempted to prove similar inequalities in a more general topological framework [88, p. 271-272].

It is not even clear that a weaker version Sys $^{2}<\left(\frac{2}{\sqrt{3}}+\epsilon\right)$ Area can be obtained in the absence of LEM.

Example 5.5. Alain Connes' theory of infinitesimals is based on nonconstructive foundational material 85 Connes wrote as follows:

Our theory of infinitesimal variables [...] will give a precise computable answer [42, p. 6207].

Connes claims that his infinitesimals provide a computable answer to a probability problem outlined earlier in his text. A constructivist who cares to analyze Connes' remarks, is faced with a stark choice: either accept the coherence of post-LEM numerical meaning as exemplified by applications of Connes' infinitesimals, or consider the founder of one of the most active fields of mathematical physics as being incoherent when talking about "computable" answers.

Example 5.6. The existence of Calabi-Yau metrics [192], influential both in differential geometry and in high-energy physics, appears to be non-constructive, as it relies on the calculus of variations, including numerous applications of versions of the extreme value theorem on several levels in infinite-dimensional spaces. In this sense, Calabi-Yau metrics pose the same problem to an anti-LEM constructivist as the Hilbert-Einstein action (see Example 5.3).

Example 5.7. A detailed study by J. Avigad [9] of ergodic theory can be viewed as an analysis of post-LEM numerical meaning, in the context of ergodic theory. More generally, the technique of proof mining seeks to extract numerical content from non-constructive proofs, using logical tools, see U. Kohlenbach and P. Oliva [120].

Foundationally speaking, the acceptance of the validity of post-LEM numerical meaning may well be a fall-back to Hilbert's position, called syntactic modeling by Avigad [9]. It is deemed acceptable to introduce non-constructive techniques, so long as one could not derive anything unacceptable from them, where "unacceptable" means numerically testable results that are found to be false 86

Attempting to analyze Hilbert's position, Stolzenberg writes as follows:

\footnotetext{
${ }^{85}$ See footnote 115 .

${ }^{86}$ Syntactic modeling may have been at the origin of Hilbert's interest in proving consistency. It is generally considered that Hilbert's hopes for a consistency proof have been dashed by Goedel's incompleteness theorems, but see Feferman [65] and Avigad and Reck [10] for a more nuanced picture.
} 
Hilbert saw formal mathematics as a way of reaching the real by passing through the ideal. That is, one may use all the formal machinery, in particular, nonconstructive but formally valid existence statements (such as the Bolzano-Weierstrass theorem), to prove, formally, real propositions, i.e. predictive ones [164].

Stolzenberg concludes on the following optimistic note:

However, not only are such considerations largely ignored or blurred nowadays, but we have already quoted, and can confirm, Bishop's observation that in practice such proofs are already constructive or can easily be made so [164].

Stolzenberg examines Hilbert's defense of classical mathematics (based as it is on classical logic, including LEM), and finds it superfluous. In our terminology introduced earlier, Stolzenberg appears to claim that post-LEM numerical meaning "can easily be made" pre-LEM. Now the constructivisation of classical results may succeed (at any rate modulo strengthening the hypotheses or weakening the conclusions, see Subsection 4.1) in the case of statements such as the intermediate value theorem (perhaps even, as implied by Stolzenberg, the BolzanoWeierstrass theorem), and the like. But Bishop apparently believed that a Robinson infinitesimal resists constructivisation.

5.3. How could an intuitionist accept the hyperreals? If both forms of constructivism discussed in Subsection 5.1 are opposed to the law of excluded middle, then wouldn't Robinson's theory run afoul of both, seeing that non-standard analysis rests on non-constructive foundations of classical logic?

To answer the question, we will start by pointing out that the burden of explanation is upon the constructivists on at least the following two counts:

(1) How is it that one of their own, A. Heyting, described Robinson's theory as not only meaningful but a standard model of important mathematical research? (see Subsection 7.3 for an analysis of Heyting's comments) 87

\footnotetext{
${ }^{87}$ Heyting gave the first formal development of intuitionistic logic in order to codify Brouwer's way of doing mathematics; yet, he unequivocally sides with the view of intuitionism as a companion, rather than alternative, to classical mathematics (see Subsection 7.3).
} 
(2) to the extent that non-standard analysis shares its foundations with, and is therefore part of, mainstream mathematics, a rejection of the former entails a radical rejectionist stance (on the part of the constructivist) that in particular encounters serious difficulties vis-a-vis important results of relativity theory, such as the Hawking-Penrose theorem, see Subsections 8.2 and 8.4.

Bishop sometimes seems to identify his notion of numerical meaning, with meaning in a lofty epistemological sense, see Section 8, but Heyting appears to view numerical meaning as a desirable, but not exclusive, methodological goal. Thus, Bishop and Heyting disagree as to the coherence of post-LEM numerical meaning (see Subsection 5.2).

Heyting's position is not inconsistent with what we described in Subsection 5.1 as numerical constructivism, see also Subsection 7.2 below. One can adopt the methodological goals of a search for greater $n u-$ merical meaning, without locking oneself into an anti-LEM constructivism 88

In Heyting's book [101, a protagonist named Class (apparently alluding to classical logic) asserts that "intuitionism should be studied as a part of mathematics". Note that a protagonist named Int (apparently alluding to intuitionism) readily agrees to Class's contention (see Subsection 7.2). Is Int merely being polite in conceding a point to Class? There is no evidence in Heyting's book that he is merely being indirect. On the other hand, one could well analyze Bishop's comment in this light. When Bishop writes [25] that he "does not know" whether non-standard analysis results in a loss of meaning, and in the same paragraph proceeds to characterize non-standard calculus as a debasement of the latter (see Section 3.1), one could feel that his comments can be described as indirect.

\section{The FERVOR OF Bishopian CONSTRUCtIVISM}

In this section, we seek to illustrate Bishop's influence on the standards of the debate concerning the nature of mathematics.

6.1. Pourciau on phlogiston. The influence of Bishop's radical philosophy has been felt in the area of discourse and standards of civility, in publications that have appeared in periodicals of the history and the philosophy of mathematics. One of the most rousing affirmations of the anti-LEM credo in English was penned by B. Pourciau and published in the Studies in History and Philosophy of Science:

\footnotetext{
${ }^{88}$ See Crowe's comment in the main text following footnote 10.
} 
The faith that sustains the classical world view emanates from one belief more than any other: that mathematical assertions are true or false, independently of our knowing which. Every conflict between classical and intuitionist mathematics springs ultimately from this belief. This is not a belief so much as a hidden cause: it creates the world view-making, for example, proofs by contradiction appear self-evidently correct-but remains transparently in the background, unseen and unquestioned [142, p. 319] [emphasis added-authors].

Such an unquestioning stance naturally leads to calcification, as Pourciau continues:

Once created, the classical world view is sustained and calcified by metaphor taken literally, by what Stolzenberg 89 calls a

"present tense" language of "objects and their properties".

Talk about sets, functions, real numbers, theorems and so on is taken by the classical mathematician as being literally about mathematical objects that exist independently of us, even though such talk, classically interpreted, has the appearance, and nothing more, of being meaningful 90 [142, p. 319] [emphasis added-authors].

The reader recognizes the pet constructivist term, meaning, already analyzed in Subsection 3.1 (see also Subsection 6.2 below), as Pourciau continues by quoting Stolzenberg as follows: "To anyone who starts off in the contemporary mathematician's belief system', argues Stolzenberg [165, p. 268],

the discovery that an entire component of the 'reality' of one's experience is produced by acts of acceptance as such in the domain of language use is not merely illuminating. In a literal sense, it is shattering. Once a mathematician has seen that his perception of the 'self-evident correctness' of the law of excluded middle is nothing more than the linguistic equivalent of an optical illusion, neither his practice of mathematics nor his understanding of it can ever be the same" [142, p. 319] [emphasis added-authors].

\footnotetext{
${ }^{89}$ See [165, p. 225].

${ }^{90}$ Pourciau's analysis of classical mathematics conflates two distinct positions, described by W. Tait [171] as realism and super-realism.
} 
Pourciau's introduction, with its talk of "conflict", "acts of acceptance", and "shattering" discoveries, is a good sample of an insurrectional narrative, two types of which are analyzed below in Subsections 6.3 and 6.4. J. Avigad, while agreeing that

$[\mathrm{w}] \mathrm{e}$ do not need fairy tales about numbers and triangles prancing about in the realm of the abstracta[,]

notes that

$[\mathrm{p}]$ roof-theoretic analysis $[\ldots]$ yields satisfying philosophical explanations as to how abstract, infinitary assumptions have bearing on computational concerns, and provides senses in which infinitary methods can be seen to have finitary content [8] 91

Having rounded off his "shattering" introduction, Pourciau now prepares his main thesis by quoting G. Giorello as follows.

One may ask whether there is a 'phlogiston 92 in mathematics. ... I would be inclined to say 'No.' This, in our opinion, would constitute a difference between a mathematical revolution and a 'revolution' in Kuhn's sense [74, p. 168].

Pourciau takes this as evidence that Giorello does not argue against the position, attributed to Crowe, that Kuhnian revolutions are "inherently impossible" in mathematics. Meanwhile, Pourciau himself argues that, had Brouwer's rejection of LEM been generally accepted, it would in fact have constituted such a revolution. Thus Pourciau appears to understand Intuitionism as viewing LEM as just such a variety of phlogiston, a position we would characterize as anti-LEM (see Subsection 5.1).

To elaborate, we argue as follows. Had Pourciau not thought of LEM as mathematical phlogiston, he would not have taken Giorello's remarks as evidence that Giorello does not argue against Crowe's contention that revolutions never occur in mathematics. The contradiction proves that Pourciau does in fact think of LEM as mathematical phlogiston. Our argument by contradiction, of course, is only meaningful classically, and would presumably be incoherent in Pourciau's preferred intuitionistic framework. However, we include it here for the benefit of a classically minded reader.

\footnotetext{
${ }^{91}$ For more details see Example 5.7 in Subsection 5.2 above.

${ }^{92}$ Phlogiston was once thought to be a fire-like element contained within combustible bodies, and released during combustion, but became incommensurable 250 years ago.
} 
Pourciau's novel view is that Brouwer's Intuitionism constituted a, failed, Kuhnian revolution. Pourciau motivates his view by dint of the observation that certain classically true assertions become "incommensurable" in Brouwer's framework. Note that the essence of Pourciau's view had already been captured by Giorello, who wrote that

"even rigorists like Weierstrass were labelled 'metaphysicians' by strict constructivists like Weyl or Brouwer" [74, p. 166].

Pourciau's main example is the (classically true) assertion to the effect that

in the infinite decimal expansion of $\pi$, either finitely many pairs of consecutive equal digits occur, or infinitely many pairs occur [142, p. 305].

Pourciau describes this assertion as becoming "incommensurable" in an intuitionistic framework. Based on the loss of such assertions in an intuitionistic framework, Pourciau concludes that Intuitionism possessed a potential of developing into a unique mathematical Kuhnian revolution. Pourciau writes that

from inside the classical paradigm [...] Kuhnian revolutions appear to be [...] obviously impossible. But from outside the classical paradigm, [...] it is no longer self-evident that Kuhnian revolutions cannot occur [142, p. 300].

He argues that both Crowe and Dauben

see Kuhnian revolutions as logically impossible, because to them [...] any shift in conceptions of mathematics must be cumulative. [142, p. 301] [emphasis in the original-the authors].

Pourciau views such cumulativity as a necessary consequence of the classical (i.e. LEM-circumscribed) paradigm, suggesting that a Kuhnian revolution in mathematics would in fact be impossible, without first extirpating LEM.

However, contrary to Pourciau's claim, mathematical entities have indeed become "incommensurable" through historical changes in mathematical practice, ever before intuitionism came on the scene.

Consider, for example, the largely successful assault on infinitesimals in the aftermath of the rise of Weierstrassian epsilontics. Cauchy's 1821 sum theorem [39] on the convergence (to a continuous limit) of a series of continuous functions under a suitable "pointwise" convergence, was declared to be false in the absence of infinitesimals (see below). 
With hindsight, we can now affirm that Cauchy's sum theorem had been made "incommensurable" in the second half of the 19th century. The sum theorem was only successfully "revived" fully a century later. A similar observation applies to infinitesimals themselves (see Example 5.2). Thus, Weierstrassian epsilontics constitute a, successful, Kuhnian revolution, by Pourciau's standard. Indeed, Robinson [148, p. 271273] proposed an interpretation of Cauchy's sum theorem that would make it correct, in the context of an infinitesimal-enriched continuum, see also J. Cleave [40, Cutland et al. [46], Goldblatt [80, p. 90], and Bråting [32]. This (ongoing) project appears to be a striking realisation of a reconstruction project enunciated by I. Grattan-Guinness, in the name of Freudenthal [72]:

it is mere feedback-style ahistory to read Cauchy (and contemporaries such as Bernard Bolzano) as if they had read Weierstrass already. On the contrary, their own pre-Weierstrassian muddles need historical reconstruction [85, p.176].93

6.2. A constructive glossary. As we already mentioned, Bishop's approach is rooted in Brouwer's revolt against the non-constructive nature of mathematics as practiced by his contemporaries 94

The Brouwer-Hilbert debate captured the popular mathematical imagination in the 1920s. Brouwer's crying call was for the elimination of most of the applications of LEM from meaningful mathematical discourse. Burgess discusses the debate briefly in his treatment of nominalism in [37, p. 27]. We analyzed Bishop's implementation of Brouwer's nominalistic project in [111.

Bishop's program has met with a certain amount of success, and attracted a number of followers. Part of the attraction stems from a detailed lexicon developed by Bishop so as to challenge received (classical) views on the nature of mathematics. A constructive lexicon was a sine qua non of his success. A number of terms from Bishop's constructivist lexicon constitute a novelty as far as intuitionism is concerned, and are not necessarily familiar even to someone knowledgeable about intuitionism per se. It may be helpful to provide a summary of such terms for easy reference, arranged alphabetically, as follows.

\footnotetext{
${ }^{93}$ See Borovik \& Katz [1] and Katz \& Katz 112 for a detailed analysis of trends in Cauchy historiography.

${ }^{94}$ Similar tendencies on the part of Wittgenstein were analyzed by H. Putnam, who describes them as "minimalist" [143, p. 242]. See also G. Kreisel [82].
} 
- Debasement of meaning is the cardinal sin of the classical opposition, from Cantor to Keisler 95 committed with LEM (see below). The term occurs in Bishop's Schizophrenia [27] and Crisis [25] texts.

- Fundamentalist excluded thirdist is a term that refers to a classicallytrained mathematician who has not yet become sensitized to implicit use of the law of excluded middle (i.e., excluded third) in his arguments, see [145, p. 249] 96

- Idealistic mathematics is the output of Platonist mathematical sensibilities, abetted by a metaphysical faith in LEM (see below), and characterized by the presence of merely a peculiar pragmatic content (see below).

- Integer is the revealed source of all meaning (see below), posited as an alternative foundation displacing both formal logic, axiomatic set theory, and recursive function theory. The integers wondrously escape 97 the vigilant scrutiny of a constructivist intelligence determined to uproot and nip in the bud each and every Platonist fancy of a concept external to the mathematical mind.

In Bishop's system, the integers are uppermost to the exclusion of the continuum. Bishop rejected Brouwer's work on an intuitionstic continuum in the following terms:

Brouwer's bugaboo has been compulsive speculation about the nature of the continuum. His fear seems to have been that, unless he personally intervened to prevent it, the continuum would turn out to be discrete. [The result was Brouwer's] semimystical theory of the continuum [23, p. 6 and 10].

- Integrity is perhaps one of the most misunderstood terms in Errett Bishop's lexicon. Pourciau in his Education [141] appears to interpret it as an indictment of the ethics of the classical opposition. Yet in his Schizophrenia text, Bishop merely muses:

I keep coming back to the term "integrity". [27, p. 4]

\footnotetext{
${ }^{95}$ But see footnote 103 .

${ }^{96}$ This use of the term "fundamentalist excluded thirdist" is in a text by Richman, not Bishop. I have not been able to source its occurrence in Bishop's writing. In a similar vein, an ultrafinitist recently described this writer as a "choirboy of infinitesimology"; however, this term does not seem to be in general use. See also Subsections 114 and 8.1

${ }^{97} \mathrm{By}$ dint of a familiar oracular quotation from Kronecker; see also main text around footnote 48
} 
Note that the period is in the original. Bishop describes integrity as the opposite of a syndrome he colorfully refers to as schizophrenia, characterized by a number of ills, including

(a) a rejection of common sense in favor of formalism,

(b) the debasement of meaning (see above),

(c) as well as by a list of other ills-

but excluding dishonesty. Now the root of

$$
\text { integr-ity }
$$

is identical with that of integer (see above), the Bishopian ultimate foundation of analysis. Bishop's evocation of integrity may have been an innocent pun intended to allude to a healthy constructivist mindset, where the integers are uppermost.

Brouwer sought to incorporate a theory of the continuum as part of intuitionistic mathematics, by means of his free choice sequences. Bishop's commitment to integr-ity is thus a departure from Brouwerian intuitionism.

- Law of excluded middle (LEM) is the main source of the nonconstructivities of classical mathematics 98 Every formalisation of intuitionistic logic excludes $L E M$; adding $L E M$ back again returns us to classical logic.

- Limited principle of omniscience (LPO) is a weak form of LEM (see above), involving $L E M$-like oracular abilities limited to the context of integer sequences 99 The $L P O$ is still unacceptable to a constructivist, but could have served as a basis for a meaningful dialog between Brouwer and Hilbert (see [25]), that could allegedly have changed the course of 20th century mathematics.

- Meaning is a favorite philosophic term in Bishop's lexicon, necessarily preceding an investigation of truth in any coherent discussion. In Bishop's writing, the term meaning is routinely conflated with $n u$ merical meaning (see below).

- Numerical meaning is the content of a theorem admitting a proof based on intuitionistic logic, and expressing computationally meaningful facts about the integers 100 The conflation of numerical meaning with meaning par excellence in Bishop's writing, has the following two consequences:

\footnotetext{
${ }^{98}$ See Example 2.1

${ }^{99}$ See Subsection 3.1 for a discussion of LPO.

${ }^{100}$ As an illustration, a numerically meaningful proof of the irrationality of $\sqrt{2}$ appears in Example 2.1.
} 
(1) it empowers the constructivist to sweep under the rug the distinction between pre-LEM and post-LEM numerical meaning 101 lending a marginal degree of plausibility to a dismissal of classical theorems which otherwise appear eminently coherent and meaningful, 102 and

(2) it allows the constructivist to enlist the support of anti-realist philosophical schools of thought (e.g. Michael Dummett) in the theory of meaning, inspite of the apparent tension with Bishop's otherwise realist declarations (see entry Realistic mathematics below).

- Peculiar pragmatic content is an expression of Bishop's [23, p. viii] that was analyzed by Billinge [21, p. 179]. It connotes an alleged lack of empirical validity of classical mathematics, when classical results are merely inference tickets [21, p. 180] used in the deduction of other mathematical results.

- Realistic mathematics. The dichotomy of "realist" versus "idealist" (see above) is the dichotomy of "constructive" versus "classical" mathematics, in Bishop's lexicon. There are two main narratives of the Intuitionist insurrection, one anti-realist and one realist. The issue is discussed in Subsections 6.3 and 6.4.

On the first page of his book, Bishop claims that constructive mathematics based on the natural numbers will still be the same, "in another universe, with another biology and another physics" [23, p. 1]. Since the sensory perceptions of the human body are physics- and chemistrybound, a claim of such trans-universe invariance amounts to the positing of a disembodied nature of the natural number system (transcending the physics and the chemistry). Bishop's disembodied natural numbers are the cornerstone of his approach.

6.3. Insurrection according to Michael. The anti-realist narrative, mainly following Michael Dummett [60], traces the original sin of classical mathematics with $L E M$, all the way back to Aristotle 103 The law of excluded middle (see Subsection 6.2) is the mathematical counterpart of geocentric cosmology (alternatively, of phlogiston, see

\footnotetext{
${ }^{101}$ See Subsection 5.2

${ }^{102}$ See Subsection 4.3 for a discussion of the extreme value theorem in an intuitionstic context.

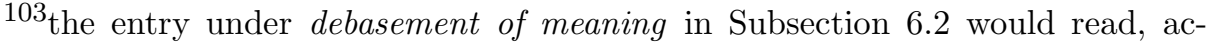
cordingly, "the classical opposition from Aristotle to Keisler"; see main text at footnote 95 .
} 
Subsection 6.1), slated for the dustbin of history 104 The anti-realist narrative dismisses the Quine-Putnam indispesability thesis (see Subsection 8.2), on the grounds that a philosophy-first examination of first principles is the unique authority empowered to determine the correct way of doing mathematics 105 Generally speaking, it is this narrative that seems to be favored by a number of philosophers of mathematics.

Dummett opposes a truth-valued, bivalent semantics, namely the notion that truth is one thing and knowability another, on the grounds that it violates Dummett's manifestation requirement, see Shapiro [160, p. 54]. The latter requirement, in the context of mathematics, is a restatement of the intuitionistic principle that truth is tantamount to verifiability. Thus, an acceptance of Dummett's manifestation requirement, leads to intuitionistic semantics and a rejection of LEM.

In his foundational text [59] originating from 1973 lecture notes, Dummett is frank about the source of his interest in the intuitionist/classical dispute in mathematics:

This dispute bears a strong resemblance to other disputes over realism of one kind or another, that is, concerning various kinds of subject-matter (or types of statement), including that over realism about the physical universe 106 , p. ix] [emphasis added-authors].

What Dummett proceeds to say at this point, reveals the nature of his interest:

but intuitionism represents the only sustained attempt by the opponents of a realist view to work out a coherent embodiment of their philosophical beliefs [emphasis added-authors].

What interests Dummett here is the fight against the realist view. What endears intuitionists to him, is the fact that they have succeeded where the phenomenalists have not:

Phenomenalists might have attained a greater success if they had made a remotely comparable effort to show in detail what consequences their interpretation of materialobject statements would have for our employment of our language [59, p. ix].

\footnotetext{
${ }^{104}$ Following Kronecker and Brouwer, Dummett rejects actual infinity, at variance with Bishop.

${ }^{105}$ See discussion of the views of Billinge and Hellman in the main text at footnote 79 .

${ }^{106}$ The strong resemblance claim is precisely the point we wish to dispute, see below.
} 
However, Dummett's conflation of the mathematical debate and the philosophical debate, could be challenged.

We hereby explicitly sidestep the debate opposing the realist (as opposed to the super-realist, see W. Tait [171]) position and the antirealist position. On the other hand, we observe that a defense of indispensability of mathematics would necessarily start by challenging Dummett's manifestation requirement. More precisely, such a defense would have to start by challenging the extension of Dummett's manifestation requirement, from the realm of philosophy to the realm of mathematics. While Dummett chooses to pin the opposition to intuitionism, to a belief in an

interpretation of mathematical statements as referring to an independently existing and objective reality [59, p. ix],

(i.e. a Platonic world of mathematical entities), J. Avigad memorably retorts as follows:

We do not need fairy tales about numbers and triangles prancing about in the realm of the abstracta [8].

A. Weir writes that Dummett's argument hinges on questionable empiricist dogma:

Dummett claims that no teacher could manifest and communicate to a pupil, about to acquire a language, grasp of sentences which are determinately true or false though we may be incapable of determining which. But the argument seems to hinge on the empiricist dogma that what is learned cannot radically transcend the experiential or stimulatory inputs to learning [186, p. 465].

6.4. Insurrection according to Errett. Turning now to the realist narrative of the intuitionist insurrection, we note that such a narrative appears to be more consistent with what Bishop himself actually wrote. In his foundational essay, Bishop expresses his position as follows:

As pure mathematicians, we must decide whether we are playing a game, or whether our theorems describe an external reality 107 [25, p. 507].

The right answer, to Bishop, is that they do describe an external reality 108 The dichotomy of "realist" versus "idealist" is the dichotomy of

\footnotetext{
${ }^{107}$ This remark of Bishop's has been reflected in secondary sources; see 47 , p. 188].

${ }^{108}$ Our purpose here is not to endorse or refute Bishop's views on this point, but rather to document his actual position, which appears to diverge from Dummett's.
} 
"constructive" versus "classical" mathematics, in Bishop's lexicon (see entry under idealistic mathematics in Section [6.2). In [27, p. 4], Bishop mentions his ambition to incorporate such mathematically oriented disciplines as physics as part of his constructive revolution, revealing a recognition, on his part, of the potency of the Quine-Putnam indispensability challenge. 109

N. Kopell and G. Stolzenberg, close associates of Bishop, published a three-page Commentary [123] following Bishop's Crisis text. Their note places the original sin with $L E M$ at around 1870 (rather than Greek antiquity), when the "flourishing empirico-inductive tradition" began to be replaced by the "strictly logico-deductive conception of pure mathematics" 110 Kopell and Stolzenberg don't hesitate to compare the empirico-deductive tradition in mathematics, to physics, in the following terms:

[Mathematical] theories were theories about the phenomena, just as in a physical theory [123, p. 519].

Similar views have been expressed by D. Bridges, see e.g. [36], and, as we argue in Subsection 7.2 , by Heyting.

6.5. Richman on large cardinals. The Interview with a constructive mathematician was published by leading constructivist F. Richman in 1996. In the Interview, Richman seems to reject any alternative to an anti-LEM constructivism, in his very first comment:

the constructive mathematician dismisses classical mathematics as an exercise in formal logic, much like investigating the consequences of large cardinal axioms [145, p. 248].

It should be noted that fellow constructivist D. Bridges 35] appears to distance himself somewhat from Richman's Interview, which he described in 1997 as

a fascinating, provocative, and by no means standard view on constructivism in mathematics 111

\footnotetext{
${ }^{109}$ Billinge [20, p. 314] purports to detect "inchoate" anti-realist views in Bishop's writings, but provides no constructive proof of their existence, other than a pair quotes on numerical meaning. Meanwhile, Hellman writes: "Some of Bishop's remarks (1967) suggest that his position belongs in [the radical] category" 95, p. 222].

${ }^{110}$ The latter term may be referring to the Fregean revolution in logic, see footnote 116 .

${ }^{111}$ Philosophers of science such as Hellman (see [95, p. 222]) and Billinge see Brigdes as being closer to the liberal outlook, and further from the radical one,
} 
In the context of the exchange between Class and Int (see Subsection 7.2), note that Richman seems to concede a point to Class in the following group theory remark, somewhat parallel to our Pappus analogy ${ }^{112}$ in Section 7.2 .

The richness of constructive mathematics lies in the fact that concepts that are equivalent in the presence of the law of excluded middle, need not be equivalent. This is typical of generalizations: the notion of a normal subgroup is equivalent to that of a subgroup in the context of the commutative law [145, p. 257].

Yet he quickly retreats to the safety of anti-LEM, observing that

the law of excluded middle obliterates the notion of positive content $[\ldots]$

Anti-LEM constructivist examination of the foundations is typically laced with anti-theological sarcasm, as when a mainstream mathematician is described as "a fundamentalist excluded-thirdist" [145, p. 249] (see [145, p. 260] for additional deistic sarcasm), betraying a nonnegotiable commitment to an ideology 113 Yet we detect a glimmer of hope here, when Richman writes:

It now seems apparent to me-although I did not realize this for many years-that for all practical purposes, constructive mathematics coincides with mathematics done in the context of intuitionistic logic [145, p. 253] [emphasis added-authors].

While little evidence is offered for such a counter-intuitive (no pun intended) assertion, it sets out the following hope. If a numerical constructivist can, after many years, be led to abandon numerical constructivism and switch to anti-LEM; then also, at some future time, anti-LEM constructivists can perhaps be persuaded, by force of overwhelming evidence, to abandon anti-LEM and switch to numerical constructivism of the companion variety. The nature of such evidence will be discussed below.

than Richman, whether or not Bridges meant this comment as a way of distancing himself from Richman.

${ }^{112}$ See discussion at footnote 119 .

${ }^{113}$ Whether or not Richman meant this comment to be tongue-in-cheek, constructivists are merely following Bishop's sarcastic lead, see [23, p. 2] as well as Bishop's "diabolical" limerick entitled Formalism, characterizing the latter as "sawdust", while constructivism as "the heart" [27, p. 14]. 
Richman's disillusionment with classical mathematics was triggered by a perceived lack of meaning in uncountable, torsion abelian group theory. Richman writes that, even in the countable case,

the centerpiece of the subject, the classification theorem for countable torsion abelian groups, cannot even be stated without ordinal numbers [144].

To Richman, the non-constructivity of the arguments appears to be compounded by the non-constructive formulation of the very statements of the results of torsion abelian group theory. Richman apparently perceived a lack of numerical meaning of even the post-LEM kind, as discussed in Subsection 5.2. If Richman's disillusionment with classical mathematics was predicated on a conflation of the two types of numerical meaning, then it leaves open the possibility for other constructivists to sympathize with Richman's disillusionment with torsion abelian groups, without, however, jettisoning post-LEM numerical meaning of classical mathematics 114

What is the nature of the evidence in favor of a numerical constructivism of a companion variety? A mathematician working in the tradition of Archimedes, Leibniz, and Cauchy owes at least a residual allegiance to the idea that the most important mathematical problems are those coming from physics, engineering, and science more generally. A mathematical theory that has wide-ranging applications, be it Abraham Robinson's infinitesimals or Alain Connes' infinitesimals, 115 is not

\footnotetext{
${ }^{114}$ See also footnote 81

${ }^{115}$ A. Connes criticizes Robinson's infinitesimals for being dependent on nonconstructive foundational material. He further claims it to be a weakness of Robinson's infinitesimals that the results of calculations that employ them, do not depend on the choice of the infinitesimal. Yet, Connes himself develops a theory of infinitesimals bearing a striking similarity to the ultrapower construction of the hyperreals. Furthermore, he freely relies on such results as the existence of the Dixmier trace, and the Hahn-Banach theorem. The latter results rely on similarly nonconstructive foundational material. Connes claims the independence of the choice of Dixmier trace to be a strength of his theory of infinitesimals in [42, p. 6213]. Thus, both of Connes' criticisms apply to his own theory of infinitesimals. As far as the introduction of non-commutativity by Connes is concerned, it is similar to the enlargement from the diagonal matrices, to the spectrum of arbitrary symmetric matrices. Such an enlargement is a natural generalisation. Its commutative antecedent cannot be criticized for commutativity, any more than one can criticize the Cartan subalgebra of, say, a matrix algebra, for being abelian. The mathematical novelty of Connes' theory of infinitesimals resides in the exploitation of Dixmier's trace, relying as it does on non-constructive foundatoinal material, thus of similar foundational status to, for instance, the ultrapower construction of a non-Archimedean extension of the reals. See also Example 5.5 above.
} 
easily discounted as meaningless, even if its starting point may rely on foundational material that is not fully constructive. This thread is pursued in more detail in Section 8 .

\section{IS CONSTRUCTIVE MATHEMATICS PART OF CLASSICAL MATHEMATICS?}

7.1. From Cantor and Frege to Brouwer. W. Tait [170] argues that, unlike intuitionism, constructive mathematics is part of classical mathematics. We would like to go further, and suggest that Frege's revolutionary logic (see Gillies [75]) and Cantor's revolutionary set theory (see Dauben [48]) created a new language and a new paradigm, transforming mathematical foundations into fair game for further investigation, experimentation, and criticism, including intuitionistic criticism voiced by Brouwer 116

Classical mathematics as pursued by Frege and Cantor literally created a universe of discourse where Brouwer's insurrectional narrative could be articulated and enjoy a hearing. In this sense, intuitionism is not a rival, but an offspring, of classical mathematics. To quote J. Avigad,

[t]he syntactic, axiomatic standpoint has enabled us to fashion formal representations of various foundational stances, and we now have informative descriptions of the types of reasoning that are justified on finitist, predicative, constructive, intuitionistic, structuralist, and classical grounds [8].

This idea, as applied to intuitionism, was expressed by J. Gray in the following terms:

Intuitionistic logics were developed incorporating the logical strictures of Brouwer; constructivist mathematics still enjoys a certain vogue. But these are somehow

\footnotetext{
${ }^{116}$ Cantor's set theory is familiar to a general mathematical audience, and requires no special comment. The revolutionary nature of Frege's work from 1879, cf. [71, is widely known in logic and philosophy circles. To summarize Gillies' thesis, the Fregean revolution in logic was a change from the Aristotelian paradigm: the theory of syllogism was replaced by propositional calculus and first-order predicate calculus. The basis for the logical notation we today take for granted was in fact laid by Frege, under the name Begriffsschrift, 'conceptual notation'. (Frege himself used a 2-dimensional notation that has not been accepted by logicians, who have given preference to a linear notation more along the lines of Peano, who was strongly influenced by Frege.) For a fascinating account of the genesis of the $B e$ griffsschrift in Frege's 'Logicist program' of showing that the truths of arithmetic are analytic (rather than synthetic), see Gillies [75, p. 288].
} 


\section{contained within the larger framework of modern} mathematics 117 [87, p. 242] [emphasis added-authors].

In a similar vein, no less an authority than M. Heidegger wrote (almost simultaneously with Kolmogorov quoted in Subsection 2.2) as follows:

The level which a science has reached is determined by how far it is capable of a crisis in its basic concepts [92, p. 29-30] [emphasis added-authors].

Lest one should doubt whether Heidegger meant for his comments to apply to mathematics, he continues:

Mathematics, which is seemingly the most rigorous and most firmly constructed of the sciences, has reached a crisis in its 'foundations'.

Lest one should doubt whether he had Brouwer in mind, Heidegger continues:

In the controversy between the formalists and the intuitionists, the issue is one of obtaining and securing the primary way of access to what are supposedly the objects of this science [emphasis added-authors].

What Heidegger appears to be saying is that if we take the supposed objects to be sets or integers, the issue becomes whether a primary way of accessing them is provided by the law of excluded middle. Our interest in Heidegger's remarks stems from his observation that a science capable of such a foundational crisis, had necessarily reached an appropriate level of maturity, which we attribute to earlier developments due to Frege (via Peano), Cantor, and others.

7.2. Heyting's good right. A. Heyting gave the first formal development of intuitionistic logic in order to codify Brouwer's way of doing mathematics. Yet, we will show that Heyting unequivocally sides with the view of intuitionism as a companion, rather than alternative, to classical mathematics (see also Subsection 7.3). Heyting's example shows that a concern over the non-constructivity of the widespead use of the law of excluded middle in classical mathematics, need not necessarily lead to a radical position expressed in some of Bishop's writing.

\footnotetext{
${ }^{117}$ Gray concludes with the following thought on current debates in the foundations of mathematics: "either they are technical and accessible only to logicians, or they are epistemological and draw their examples from concepts we meet in school". Examples going beyond the concepts we meet in school are discussed in Subsections 5.2 and 8.4
} 
A. Heyting's book Intuitionism [101] dates from 1956. The (first part of the) book is written in the form of a dialog among representatives of some of the main schools of thought in the foundations of mathematics. We will now analyze Heyting's comments in more detail. The protagonist named Class makes the following point:

Intuitionism should be studied as part of mathematics. In mathematics, we study consequences of given hypotheses. The hypotheses assumed by intuitionists may in fact be interesting, but they have no right to a monopoly [101, p .4] [emphasis added-authors].

In other words, emptying our logical toolkit of the law of excluded middle is one possible foundational framework among others 118 This could be compared, for example, to studying projective geometry with Pappus' axiom eliminated 119 Class's claim appears to be accepted by the protagonist Int. Consider Int's response to Class's remarks:

Nor do we claim that; we [i.e., intuitionists] are content if you admit the good right of our conception [emphasis added-authors].

In this connection, S. Shapiro notes:

The later Heyting did not claim a "monopoly" on mathematics, and would rest content if the classical mathematician "admits the good right of" the intuitionistic conception [160, p. 55-56].

One can legitimately pose the question whether Int is merely being polite in his response to Class. Note that later on in the same chapter, Int concedes that intuitionism has few applications, and compares it to "history, art, and light entertainment" [101, p. 10]. He does this, obviously, not as a way of disparaging intuitionism, but as a way of underscoring its intrinsic value as an intellectual pursuit, as distinct from a scientific pursuit. P. Maddy claims that

Heyting [...] is happy to dismis: 120 the loss of nonconstructive mathematics as 'the excision of noxious ornaments' [128, pp. 1121-1122],

but a closer examination of Heyting's remark [101, p. 11] reveals a more nuanced picture. Here Int is responding to Form's charge to the effect that constructivists

\footnotetext{
${ }^{118}$ See footnote 16 on the non-uniqueness of intuitionistic logic.

${ }^{119} \mathrm{~A}$ related point in the context of group theory is made by F. Richman [145, see Subsection 6.5.

${ }^{120}$ We saw above that Shapiro clearly disagrees with Maddy on this point.
} 
destroy the most precious mathematical results [101, p. 10].

Int replies as follows:

As to the mutilation of mathematics of which you accuse me, it must be taken as an inevitable consequence of our standpoint. It can also be seen as an excision of noxious ornaments, $[\ldots]$ and it is at least partly compensated for by the charm of subtle distinctions and witty methods by which intuitionists have enriched mathematical thought [emphasis added-authors].

His comments show a clear recognition of the potency of the mutilation challenge, as well as a necessity to compensate for the damages.

Heyting's position is not inconsistent with a numerical constructivism, as opposed to an anti-LEM constructivism, a kind of a long march through the foundations launched in sixty-seven [23]. Heyting was able to, so to speak, rise above differences of Class and Int.

What would Heyting have thought of a rigorous justification of infinitesimals in the framework of classical logic? Remarkably, we have a detailed testimony by Heyting himself as to the respect he bore for Robinson's accomplishment, see Subsection 7.3. Already in 1956, Heyting allowed his protagonist Form to cite a reference by A. Robinson [146] in a comment dealing with

the use of metamathematics for the deduction of mathematical results [101, p. 45].

In fact, in 1973, Heyting compared Robinson's work favorably with that of Brouwer himself, according to a Math Reviews summary:

[Heyting] gives a brief description of the nature of Robinson's work in model theory and reflects on how that work resembles that of Brouwer and thereby qualifies for the award of the Brouwer medal [102].

S. Shapiro [160, in a retort to N. Tennant 178, unveils an allegedly irreducible clash between classical mathematics, on the one hand, and intuitionism as expressed through Heyting's semantics, on the other. Here Heyting semantics refers to the constructive interpretation of the quantifiers discussed in Subsection 2.3. Shapiro writes as follows:

Let $\chi(x)$ be any predicate that applies to natural numbers. It is a routine theorem of classical arithmetic that

$$
\forall x \exists ! y[(\chi(x) \wedge y=0) \vee(\neg \chi(x) \wedge y=1)]
$$

(Shapiro [160, p. 60]). 
Classically, Shapiro's formula merely asserts that $\chi(x)$ is either true or false (where $y$ is assigned respectively the value 0 or 1 ). Shapiro continues:

Under Heyting semantics, this proof amounts to a thesis that there is a computable function that decides whether $\chi$ holds. So under Heyting semantics [...] every predicate is effectively decidable[, which is] a tough pull to swallow and keep down 121

Namely, the constructive existence of $y$ necessarily entails being able to find a computational procedure capable of identifying such a $y$ as a function of $x$, and hence a decision procedure for the predicate $\chi$.

Does this prove that classical mathematics and intuitionism cannot get along, as Shapiro puts it? Yes, if by "classical mathematics" is meant "all of classical mathematics", down to the very last predicate. On the other hand, the answer is no, if we are to adopt Bishop's 1975 program [25], involving a comprehensive sifting of classical material.

In fact, the philosophical exchange between Tennant and Shapiro has an uncanny parallel - a quarter century earlier, in the exchange between Bishop and Timothy G. McCarthy, writing for Math Reviews [132] in 1975. In the context of Bishop's proposal to translate absolute classical statements into relative assertions of the form "LPO $\rightarrow$ such-and-such", McCarthy writes as follows:

There is an ambiguity about just what the author intends to explicate by means of the suggested translation. It is not plausible to suppose that $A$ is classically true (false) if and only if [the proposition] LPO $\rightarrow A$ ([respectively,] LPO $\rightarrow \neg A$ ) is constructively provable, in view of Gödel's first incompleteness theorem.

It appears that Bishop's intention was not to capture all classical statements, but rather to undertake a comprehensive sift. Such seems to be the position adopted by the mature Heyting, as well, as we analyze in the next section.

7.3. Heyting's Address to Professor Robinson. Intuitionist Heyting's comments in Address to Professor A. Robinson [102] are fascinating and worth reproducing in some detail:

\footnotetext{
${ }^{121} \mathrm{~A}$ similar argument involving the introduction of an auxiliary bivalent variable occurs in the proof of the Goodman-Myhill theorem, see footnote 74.
} 
(1) (p. 135) Brouwer was right that intuitionistic mathematics is the only form of mathematics which has a perfectly clear interpretation [...] and it is desirable that as much of mathematics as possible will be made constructive.

(2) (ibid.) the curious mixture of formal reasoning and more or less vague intuitions which classical mathematics is, remains an imposing work of art and [at] the same time the powerful tool in the struggle for life of mankind 122

(3) (ibid.) intuitionistic mathematics is no longer isolated from classical mathematics [emphasis added-authors].

(4) (ibid.) several mathematicians work on the foundations of intuitionistic as well as of classical mathematics.

(5) (ibid.) The two subjects become more and more intertwined.

In a remarkable tribute to Robinson's oeuvre, Heyting writes:

In your work on the metamathematics of algebra you explored systematically the connections between formal logic and algebra, firstly in the form of so-called transfer principles, by which a theorem, proved for some algebraic system, holds automatically for a whole class of such systems [102, p. 135] [emphasis added-authors].

For a discussion of the transfer principle of non-standard analysis, see Appendix A. Heyting does not stop there, and makes the following additional points:

(1) (p. 136) the creation of non-standard analysis is a standard model of important mathematical research.

(2) (ibid.) you connected this extremely abstract part of model theory with a theory apparently so far apart as the elementary calculus [emphasis added-authors].

(3) (ibid.) In doing so you threw new light on the history of the calculus by giving a clear sense to Leibniz's notion of infinitesimals.

(4) (ibid.) infinitesimals [...] are still commonly used in many fields where their elimination $[\ldots]$ would lead to unwelcome complications.

(5) (ibid.) This is the case in differential geometry and in many parts of applied mathematics such as hydrodynamics and electricity theory, where physicists use infinitesimals without a twinge of conscience.

\footnotetext{
${ }^{122}$ The curious mixture was the subject of Crowe's musings cited in the main text preceding footnote 10
} 
(6) (ibid.) You showed how these theories can be made precise by your method. Dirac's $\delta$-function is an extreme example. This mysterious object became lucid in the light of non-archimedean analysi: 123 [emphasis added-authors].

On the subject of whether there is meaning after model theory, Heyting has this to say:

(1) (p. 136) The notion of a non-standard model is complicated and highly inconstructive [sic], but its properties, though surprising, are rather simple and can therefore be understood by people who know very little about model theory;

(2) (ibid.) Once you had shown by the paradigm of the calculus how it can be used, many other applications were found by yourself as well as by many other mathematicians.

(3) (p. 137) Model theory was in our time one of the abstract theories which, so to say, floated in the air. In a sense your work can be considered as a return from this abstraction to concrete applications [emphasis added-authors].

(4) (ibid.) The general non-constructive theory of non-standard models links its applications together into a harmoni[ous] whole.

Heyting's final comment is particularly revealing. He recognizes that, while the model may be non-constructive, its applications may indeed be concrete (in the terminology of Subsection 5.2, such applications possess post-LEM numerical meaning). Such a thesis is consonant with what we called numerical constructivism in Subsection 5.1.

In Heyting's case, an acute awareness of the shortcomings of LEMdependent mathematical investigations went hand-in-hand with an appreciation of the accomplishments of classical mathematics in general, and Robinson's mathematics in particular. Such an awareness is therefore not at odds with a recognition of the coherence of post-LEM numerical meaning. It is at odds, however, with an anti-LEM radical constructivist stance, as enunciated by E. Bishop.

\section{Constructivism, Physics, And the ReAl World}

In this section we deal with challenges to anti-LEM constructivism stemming from natural science.

8.1. Bishop's view. Bishop [27, p. 4] speaks in favor of a goal of seeking

\footnotetext{
${ }^{123}$ Infinitesimal constructions of "Dirac" delta functions originate with Fourier and Cauchy, see Borovik \& Katz [31].
} 
common ground in the researches of pure mathematics, applied mathematics, and such mathematically oriented disciplines as physics [...] [emphasis added-authors].

To what extent is such a commendable goal served well by constructivism?

In the previous section, we mentioned the constructivist's problem of accounting for applications of LEM-dominated mathematics. This point seems to have bothered the interviewer of Richman's Interview:

Here is a question that has bothered me ever since the first time I read Bishop. In what sense can your [constructive] real line be used as a model for either space or time in physics? [145, p. 252]

Richman replies as follows:

I don't know exactly how to respond to the [...] question, given that I have already rejected the idea that constructivists have a real line that is different from the classical real line.

It needs to be understood what Richman meant by this cryptic remark. For convenience, we will discuss the circle $\mathbb{R} / \mathbb{Z}$ instead of $\mathbb{R}$ itself. The circle $\mathbb{R} / \mathbb{Z}$ is constructively diffeomorphic (via the exponential map) to the unit circle in the plane. A classical mathematician believes that the circle can be decomposed as the disjoint union of the lower halfcircle (with one endpoint included, and the other excluded) and its antipodal image (upper halfcircle). Meanwhile, the constructivist believes that it is impossible to decompose the circle as the disjoint union of a pair of antipodal sets. Aren't these different lines? In a similar vein, a typical physicist possesses a simple faith in the existence of infinitesimals in our familiar number system, and Komkov [121] explicitly endorses Robinson's infinitesimals. As we have documented in the present text, Bishop and his followers are less eager to accept Robinson's infinitesimals as part of our number system. Aren't these different "real lines"?

At any rate, Richman's reply above 124 does not appear to deal with the issue of scientific applications as a litmus test of the relevance

\footnotetext{
${ }^{124}$ Now an anti-LEM constructivist may be satisfied with a dodge, but the rest of us may wonder why Richman's 24-page Interview has no bibliography, particularly in view of its publication in a journal that describes itself as "an organ for rapid, low-cost, communication between historians of logic and research logicians". Did history start with E. Bishop?
} 
of mathematical production. Such an issue is related to the QuinePutnam indispensability thesis, see Feferman [66, Section IIB], M. Colyvan [41], or G. Hellman [94], see Subsection 8.2 below.

In fact, Bishop himself was challenged on the relationship to physics by $\mathrm{G}$. Mackey, in the following terms:

Consider the foundational question in physics: what is the real mathematics that the physicists are doing? [25, p. 515]

In his reply, Bishop falls back on the issue of mathematical rigor:

physicists have told me that the sort of meaning that is appropriate to physics is not to ask whether the mathematics in question is rigorous. Rather, it involves the relation of the results to the real world [emphasis addedauthors].

At first glance, Bishop's comment may seem to amount to an unobjectionable observation concerning a higher standard of rigor in mathematics. However, what Bishop is emphasizing in his essay is a notion of meaning which amounts to, as Hellman puts it, a philosophical principle of cognitive significance [96, p. 239]. Such a notion must, in Bishop's view [25, p. 509], supercede and precede a notion of truth, as well as the related one of rigor.

The kind of (unabashedly LEM-dominated) mathematics that a physicist practices is, as Bishop seems to admit matter-of-factly, successful in relating to the real world, and therefore apparently meaningful. Such a situation apparently creates a tension with Bishop's attempted identification of meaning with numerical meaning, that can only be resolved in the context of a numerical constructivism, a companion to classical mathematics, recognizing the coherence of post-LEM numerical meaning (see Subsection 5.2).

In his Constructivist manifesto, Bishop wrote that

Weyl, a great mathematician who in practice suppressed his constructivist convictions, expressed the opinion that idealistic [i.e. classical] mathematics finds its justification in its applications in physics [23, p. 10].

Bishop does not elaborate as to why he feels H. Weyl was off the mark here; this point will be discussed in Subsection 8.2.

8.2. Hellman on indispensability. The challenge to constructivism stemming from the Quine-Putnam indispesability thesis has been extensively pursued by G. Hellman. Hellman expresses an appreciation of the foundational significance of constructivism in the following terms: 
A turning point $[\ldots]$ came in the 1960s with the appearance of Erret[t] Bishop's Foundations of constructive analysis (1967) which succeeded in constructivizing large portions of classical functional analysis of just the sort used in the physical sciences (including the theory of operators on Banach and Hilbert spaces, and even measure theory) [94, p. 457].

As to the question whether Bishop's constructivism can ultimately serve the needs of the natural sciences, Hellman analyzes what he sees as limitations of constructivism in scientific applications. Hellman 995, p. 234] invokes Plato's parable of the cave, apparently reflecting his global perception of constructivism as an intellectual house of cards. However, one can recognize the coherence of the intuitionist critique of the foundations of mathematics, while rejecting the intellectual underpinnings of its insurrectional narrative, in its mutually contradictory (realist and anti-realist) versions, as discussed in Subsection 6.3.

In the context of general relativity theory, Hellman argues that, very likely, the Hawking-Penrose singularity theorems for general relativistic spacetimes are essentially non-constructive (Hellman in [98]), see also (Billinge [20]), see Subsection 8.4.

The philosopher of mathematics M. Dummett rejects outright the idea that "empirical discoveries" or applications should have any bearing on the outcome of the debate opposing constructivism and classical mathematics:

Intuitionists are engaged in the wholesale reconstruction of mathematics, not to accord with empirical discoveries, not to obtain more fruitful applications, but solely on the basis of philosophical views concerning what mathematical statements are about and what they mean [...] intuitionism will never succeed in its battle against rival, and more widely accepted, forms of mathematics unless it can win the philosophical battle 60, p. viii] [emphasis added-authors].

The battle imagery is typical of the anti-realist type of insurrectional narrative, already discussed in Subsection 6.3.

Meanwhile, the philosopher of mathematics G. Hellman holds, just as categorically, that such a philosophy-first attempt is "doomed" 125 Awaiting the outcome of the philosophical battle, we note that Dummett's stated intuitionist position qualifies for the anti-LEM species.

\footnotetext{
${ }^{125}$ See footnote 79 for Hellman's view.
} 


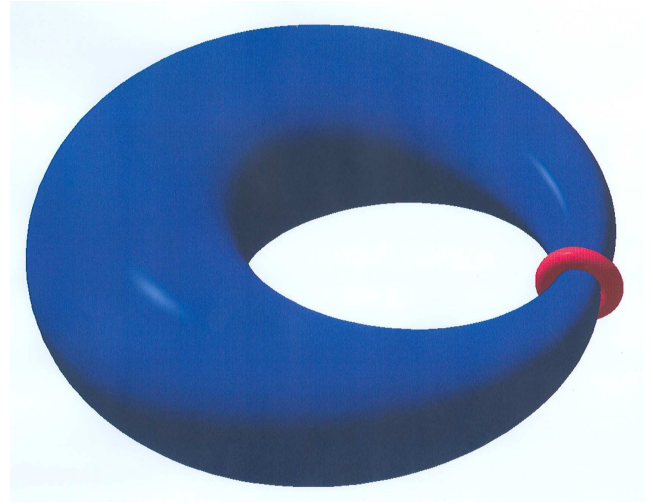

Figure 8.1. A shortest loop on a torus

More generally, variational problems tend to be resistant to efforts at constructivizing, a point apparently acknowledged by Beeson when he writes that

Calculus of variations is a vast and important field which lies right on the frontier between constructive and nonconstructive mathematics [13, p. 22].

The problem is that the extreme value theorem is not available in the absence of the law of excluded middle. The extreme value theorem is at the foundation of the calculus of variations.

As concrete examples, one could mention general existence results for geodesics, minimal surfaces, constant curvature mean surfaces, and therefore soap films and soap bubbles. It seems implausible to argue that existence results for soap films (referred to in the literature as Plateau's problem) are meaningless merely because they lack Bishopstyle numerical meaning.

Question 8.1. As a geometer, this writer would be interested in clarifying the constructive status of the following phenomenon related to Loewner's torus theorem of Example 5.4 in Subsection 5.2. Consider a rubber band "clasping" a torus in 3-space, and let it shrink to a shortest position (see Figure 8.1). Classically, one has a description of the resulting object in terms of a minimizing (possibly non-unique) closed geodesic. Providing a constructive description is not immediate, as it depends on general results of the calculus of variations, as already described above. To what extent can the existence of a loop of least length of Figure 8.1 be recaptured constructively? Pourciau's Education [141, p. 721] advocates us to "move from the simple to the less simple", but how plausible is it that such a loop could admit a simpler 
description in terms of a statement about the integers, as advocated by Integrity (see glossary in Subection 6.2)?

8.3. Novikov's perspective. S. P. Novikov's essay [138], in the "crisis" tradition of Weyl [189] and Bishop [25], deals with the crisis in the cooperation of physicists and mathematicians, in Russia and in the West. What Novikov makes excruciatingly clear is the disastrous effect of such a divorce on mathematics itself. The crucial role of variational principles is made clear already on the first page of Novikov's essay. A broad range of subjects illustrating the symbiotic relationship between the two fields can be found in Grattan-Guinness [86].

Grattan-Guinness emphasizes the role of analogies drawn from other theories, in theory-building in both mathematics and the sciences. From his viewpoint, the physicists that Novikov has in mind have been going by the book (Grattan-Guinness's book, at any rate), applying mathematical machinery by analogy, outside its original domain of applicability, and doing so with great success (in terms of predicting experiments, etc.); yet no theoretical justification for their calculations, in terms of traditional mathematics, has followed. What is worse, the gap is growing wider, according to Novikov. Traditional mathematical foundations, whether classical or intuitionist, are proving to be inadequate for the job of accounting for the progress in physics. Accordingly, Novikov is critical of set-theoretic foundations, going as far as criticizing Kolmogorov himself, for systematic efforts to introduce a set-theoretic approach in secondary education. Foundational insurrections à la Brouwer, Bishop, and Dummett (see Subsection 6.3) may amount to irrelevant distractions, from Novikov's viewpoint.

8.4. The Hawking-Penrose theorem. The Hawking-Penrose theorem in relativity theory has been the subject of something of a controversy in its own right, see G. Hellman [98], H. Billinge [20], and E. Davies [53]. Hellman argued that the Hawking-Penrose theorem is an important result which very likely does not have a constructive analog. As we explain below, Billinge's criticism of Hellman's argument is based on conflating two separate issues (geodesic incompleteness, on the one hand, and a hypothetical description of a "singularity", on the other), due to a mathematical error on her part.

Davies [53, p. 268] mentions the following points:

(1) "Hellman [98] [...] showed that the Hawking-Penrose singularity theorem is not constructively valid."

(2) "the theorem has been extremely influential in the subject."

(3) "It is embarrasssing for a radical constructivist [...]" 
(4) "It remains extremely hard to say much about the nature of the singularities [...] It is very likely that if a detailed description of the singularities becomes possible classically, that description will also be constructively valid."

We will return to Davies' remarks after reviewing the relevant mathematical details.

The Hawking-Penrose singularity theorem can be thought of as a semi-Riemannian analog of the Myers theorem [135. The latter is a result in Riemannian geometry. The result in question is a bound, modulo a suitable geometric hypothesis on the Ricci curvature, on the distance from a point to its conjugate locus. The traditional formulation of the Myers theorem relies upon the concept of a geodesic and a Jacobi field, both of which are solutions of variational problems. Myers found a bound on the distance along a geodesic $\gamma$, to the nearest vanishing point, called a conjugate point, of a Jacobi field along $\gamma$.

Returning to the Lorenzian case, note that Penrose does not even use the term singularity in formulating the result that came to be known as the Hawking-Penrose theorem. Rather, what one claims is the existence of a "past-endless geodesic [...] which has finite length" [140, p. 69]. The proof is indirect, i.e. a proof by contradiction. Penrose describes one of the main ingredients in the proof as the Raychaudhuri effect. The latter is discussed in [140, item 7.21, p. 63]. Penrose points out that

[f]or manifolds with a positive definite metric, essentially the same effect had been studied earlier by Myers (Penrose [140, p. 64]).

Thus, the formulation of the Hawking-Penrose theorem in terms of "singularities" is superfluous to its purely mathematical content, namely the fact that space-time must be (time-like or null) geodesically incomplete.

Note that there exist examples of compact Lorentz manifolds which are geodesically incomplete [91]. Penrose places singularities in quotation marks when he points out that

the physical implication of the theorem is that "singularities" (i.e., causal geodesic incompleteness) would be expected to arise whenever such a collapse takes place [140, p. 71].

(Note the significant i.e.) Whether or not geodesic incompleteness of space-time can be understood in terms of a suitable singular limiting object, boundary, or hole, is a separate question (extensively discussed 
in the physics literature, see references cited in [184, p. 213]), not germane to the problem of the foundational status (classical or intuitionist) of the Hawking-Penrose theorem.

Furthermore, while a suitable bound on the Ricci curvature does allow one to prove the existence of limiting objects in a Riemannian context (see Gromov [88, Theorem 5.3, p. 275]), such limiting objects are known to be arbitrarily pathological in general, unless one imposes additional geometric hypotheses (as in the Anderson-Cheeger theory [2], [177]), or, alternatively, leaves the domain of differential geometry and imposes conditions of algebraicity (which do not appear to be justified physically) on the manifolds being studied.

Foundationally or otherwise, finding fault with the Hawking-Penrose theorem for failing to describe a "singularity", is akin to finding fault with the Big Bang theory for failing to describe "what came before the bang".

Thus, Billinge's objection to Hellman's text [98], on the grounds of a lack of a constructive description of the singularity (echoed in item (4) above), does not appear to be based on a detailed understanding of the mathematics. The following phrase appears in Billinge:

a spacetime is singular if it is timelike or null geodesically incomplete, that is, if it has at least one timelike or null geodesic that has a point at which it comes to an end [20, p. 308] [emphasis added-authors].

The words "that is" that we have boldfaced, apparently presented by Billinge as a mathematical definition, constitute an elementary mathematical error. The spacetime in question does not contain geodesics that "come to an end", at any point of the spacetime. Rather, the geodesics are, as Penrose puts it in [140, p. 69], past-endless of finite length, i.e. they run off to (negative) infinity in finite time (in the Lorentzian case, there is no analogue of the Hopf-Rinow theorem). Naturally, if one thinks of geodesics as running into some kind of a singular "endpoint", or barrier, in spacetime, one can easily develop an impression that the relation of geodesic incompleteness to "singularities" is far more immediate than it really is.

Billinge's main source for relativity is the textbook by Wald [184]. The textbook contains the following remarks, which express the distinction between singularity and geodesic incompleteness clearly:

it is extremely difficult to give a satisfactory general notion of [a 'singularity']. We provide motivation for the notion of timelike and null geodesic incompleteness as a criterion for the presence of a singularity [...] It is this 
criterion which is used in the singularity theorems [184,

p. 212].

Furthermore, on page 214, Wald clearly states that

[u]ntil a satisfactory definition can be produced, we must

abandon the notion of a singularity as a "place".

Describing a singularity as a "place" is, of course, precisely what Billinge did at [20, p. 308]. Such an error could have been written off as a mere slip, were it not for the further evidence of insufficient background presented above.

It should be mentioned that, at variance with Davies' item (1) above, Hellman never purported to prove that "the Hawking-Penrose singularity theorem is not constructively valid", but only that he finds this "very likely", based on the non-constructive nature of the proofs found in the literature, and the general difficulties of accounting for the calculus of variations constructively (in this case, accounting for geodesics and Jacobi fields), as already discussed in Subsection 8.2 .

N. Tennant, writing on Logic, mathematics, and the natural sciences, claims that

a constructivist version of a mathematical theory is adequate for all the applications to be made of the theory within natural science [179, p. 1145].

Tennant claims that such a constructivist version is adequate for applications. Or perhaps it isn't? Tennant's claim apparently flies in the face of everything we have written here. In fact, Tennant's claim is not substantiated in his article. Tennant continues by explaining that

to be able to produce all possible refutations of empirical theories, the underlying logic can [...] be taken very weak [179, p. 1146] [emphasis added-authors].

Furthermore he concedes:

Nor do we intend to say anything about confirmation or probabilification of hypotheses by evidence [179, p. 1149].

The evidence furnished by astrophysical observation tends to confirm the hypotheses of the Hawking-Penrose theorem, but Tennant's framework does not deal with this type of scientific insight.

It is possible that, should further empirical observations one day undermine the premises of the Hawking-Penrose theorem, the resulting refutation may turn out to be adequately expressible in Tennant's version of intuitionistic logic (this does not quite follow from [179] as Tennant limits the discussion to first order logic). However, this does not alter the fact that expressing the Hawking-Penrose theorem (let 
alone proving it) remains an elusive goal, intuitionistically speaking, with the attendant loss of scientific insight provided by the theorem.

The Hawking-Penrose theorem, described by Davies as extremely influential (see item (2) above), remains a challenge to Bishopian constructivism (see item (3) above), as do the numerous variational principles mentioned by Novikov [138], see also Grattan-Guinness [86].

\section{Epilogue}

In 1973, Robinson delivered the Brouwer memorial lecture [150] on the subject of Standard and nonstandard number systems 126 J. Dauben quotes Robinson as follows:

Brouwer's intuitionism is closely related to his conception of mathematics as a dynamic activity of the human intellect $[. .$.$] This is a conception for which I$ have some sympathy and which, I believe, is acceptable to many mathematicians who are not intuitionists [50, p. 461] [emphasis added-authors].

Robinson concludes that

the dynamic evolution of mathematics is an ongoing process not only at the summit [...] but also at the more basic level of our number systems, which [tomorrow] may seem as eternal to a new generation as yesterday's technological innovations are in the eyes of a child of today.

Have today's constructivists been faithful to a conception of mathematics as a dynamic activity, as Robinson puts it? We would like to suggest that the possibility of bridging the gap between constructivism and non-standard analysis (as well as, indeed, the rest of classical mathematics) can be analyzed in the context of the dichotomy of a numerical constructivism versus an anti-LEM constructivism (see Section 5.1). Namely, only the former, consistent with Heyting's brand of intuitionism, seems sufficiently dynamic to answer the hopes [158] of reuniting the antipodes.

\section{Appendix A. Rival continua}

A Leibnizian definition of the derivative as the infinitesimal quotient

$$
\frac{\Delta y}{\Delta x}
$$

\footnotetext{
${ }^{126}$ See Heyting's introduction, quoted at length in Subsection 7.3 .
} 


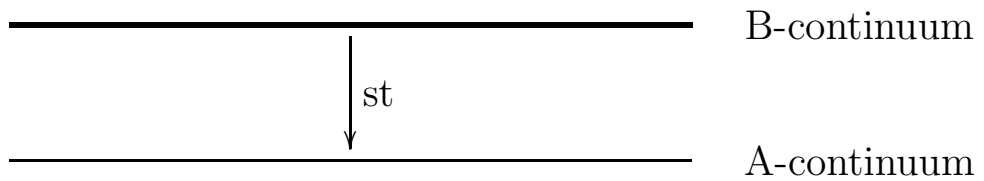

FIGURE A.1. Thick-to-thin: taking standard part (the thickness of the top line is merely conventional)

whose logical weakness was criticized by Berkeley, was modified by A. Robinson by exploiting a map called the standard part, denoted "st", from the finite part of a B-continuum (for "Bernoullian"), to the Acontinuum (for "Archimedean"), as illustrated in Figure A.1.

We will denote such a B-continuum by the new symbol $\mathbb{R}$. We will also denote its finite part, by

$$
\mathbb{R}_{<\infty}=\{x \in \mathbb{R}:|x|<\infty\},
$$

so that we have a disjoint union $\mathbb{R}=\mathbb{R}_{<\infty} \cup \mathbb{R}_{\infty}$, where $\mathbb{R}_{\infty}$ denotes the set of inverses of nonzero infinitesimals.

The map "st" sends each finite point $x \in \mathbb{R}$, to the real point $\operatorname{st}(x) \in \mathbb{R}$ infinitely close to $x$ :

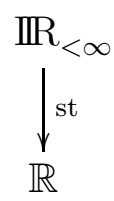

Robinson's answer to Berkeley's logical criticism 127 is to define the derivative as

$$
\text { st }\left(\frac{\Delta y}{\Delta x}\right),
$$

instead of $\Delta y / \Delta x$ as in Leibniz. "However, this is a small price to pay for the removal of an inconsistency" [148, p 266].

We illustrate the construction by means of an infinite-resolution microscope in Figure A.2.

Note that both the term "hyper-real field", and an ultrapower construction thereof, are due to E. Hewitt in 1948, see [99, p. 74]. The transfer principle allowing one to extend every first-order real statement to the hyperreals, is due to J. Eoś in 1955, see [127]. Thus, the Hewitt-Łoś framework allows one to work in a B-continuum satisfying the transfer principle. To elaborate on the ultrapower construction of

\footnotetext{
${ }^{127}$ See footnote 83 .
} 


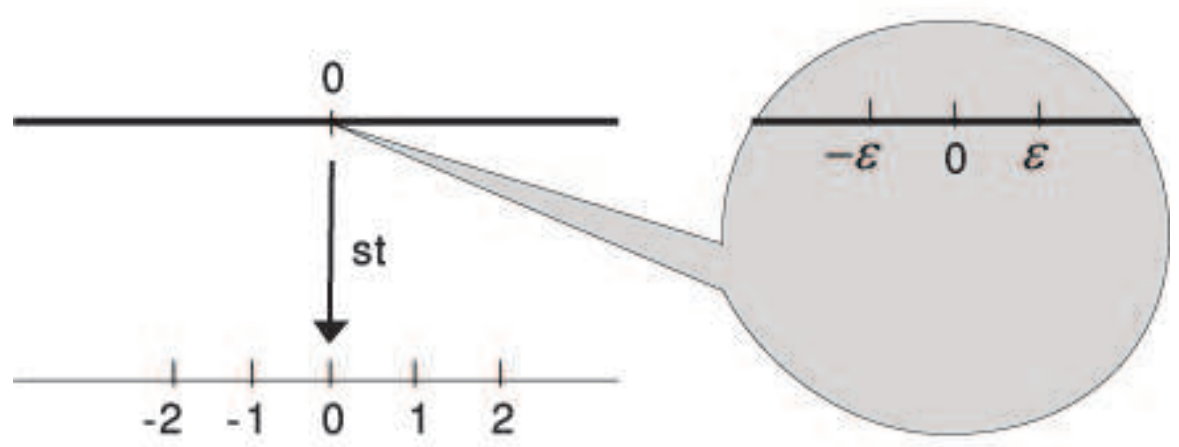

Figure A.2. Zooming in on infinitesimal $\epsilon$

the hyperreals, let $\mathbb{Q}^{\mathbb{N}}$ denote the space of sequences of rational numbers. Let $\left(\mathbb{Q}^{\mathbb{N}}\right)_{C}$ denote the subspace consisting of Cauchy sequences. The reals are by definition the quotient field

$$
\mathbb{R}:=\left(\mathbb{Q}^{\mathbb{N}}\right)_{C} / \mathcal{F}_{\text {rull }}
$$

where the ideal $\mathcal{F}_{\text {rall }}$ contains all the null sequences. Meanwhile, an infinitesimal-enriched field extension of $\mathbb{Q}$ may be obtained by forming the quotient

$$
\mathbb{Q}^{\mathbb{N}} / \mathcal{F}_{u}
$$

See Figure A.3. Here a sequence $\left\langle u_{n}\right\rangle$ is in $\mathcal{F}_{u}$ if and only if the set

$$
\left\{n \in \mathbb{N}: u_{n}=0\right\}
$$

is a member of a fixed ultrafilter 128 To give an example, the sequence $\left\langle\frac{(-1)^{n}}{n}\right\rangle$ represents a nonzero infinitesimal, whose sign depends on whether or not the set $2 \mathbb{N}$ is a member of the ultrafilter. To obtain a full hyperreal field, we replace $\mathbb{Q}$ by $\mathbb{R}$ in the construction, and form a similar quotient

$$
\mathbb{I R}:=\mathbb{R}^{\mathbb{N}} / \mathcal{F}_{u} \text {. }
$$

A more detailed discussion of the ultrapower construction can be found in M. Davis [54], Gordon et al. [83], and Giordano and Katz [79]. See also Błaszczyk [29] for some philosophical implications. More advanced properties of the hyperreals such as saturation were proved

\footnotetext{
${ }^{128}$ An ultrafilter on $\mathbb{N}$ can be thought of as a way of making a systematic choice, between each pair of complementary infinite subsets of $\mathbb{N}$, so as to prescribe which one is "dominant" and which one is "negligible". Such choices have to be made in a coherent manner, e.g., if a subset $A \subset \mathbb{N}$ is negligible then any subset of $A$ is negligible, as well. The existence of ultrafilters was proved by Tarski [176], see Keisler [118, Theorem 2.2].
} 


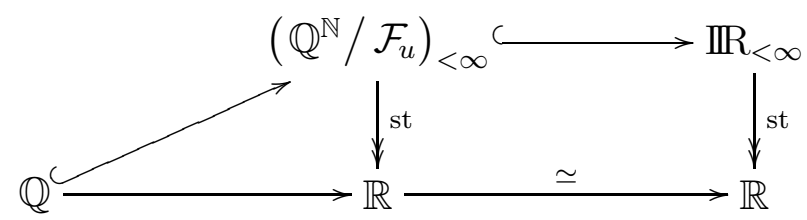

Figure A.3. An intermediate field $\mathbb{Q}^{\mathbb{N}} / \mathcal{F}_{u}$ is built directly out of $\mathbb{Q}$

later, see Keisler [117] for a historical outline. A helpful "semicolon" notation for presenting an extended decimal expansion of a hyperreal was described by A. H. Lightstone [126]. A discussion of infinitesimal optics is in K. Stroyan [168], H. J. Keisler [116], D. Tall [172], and L. Magnani and R. Dossena [129, 58]. P. Ehrlich recently constructed an isomorphism of maximal surreals and hyperreals [62]. Applications of the B-continuum range from aid in teaching calculus [64, 109, 110, 173, 175] to the Bolzmann equation (see L. Arkeryd [4, 5]); modeling of timed systems in computer science (see H. Rust [156]); mathematical economics (see Anderson [3]); mathematical physics (see Albeverio et al. [1]); etc.

In 1990, Hewitt reminisced about his

efforts to understand the ring of all real-valued continuous [not necessarily bounded] functions on a completely regular $T_{0}$-space. I was guided in part by a casual remark made by Gel'fand and Kolmogorov (Doklady Akad. Nauk SSSR 22 [1939], 11-15). Along the way I found a novel class of real-closed fields that superficially resemble the real number field and have since become the building blocks for nonstandard analysis. I had no luck in talking to Artin about these hyperreal fields, though he had done interesting work on real-closed fields in the 1920s. (My published "proof" that hyperreal fields are real-closed is false: John Isbell earned my gratitude by giving a correct proof some years later.) [...] My ultra-filters also struck no responsive chords. Only Irving Kaplansky seemed to think my ideas had merit. My first paper on the subject was published only in 1948 100

(Hewitt goes on to detail the eventual success and influence of his 1948 text). Here Hewitt is referring to Isbell's 1954 paper [105], proving that Hewitt's hyper-real fields are real closed. Note that a year later, 
Łoś [127] proved the general transfer principle for such fields, implying in particular the property of being real closed, the latter being firstorder.

\section{ACKNOWLEDGMENTS}

We are grateful to Geoffrey Hellman for insightful comments that helped improve an earlier version of the manuscript, to Douglas Bridges for a number of helpful remarks, and to Claude LeBrun for expert comments on the Hawking-Penrose theorem. Hilton Kramer's influence is obvious throughout.

\section{REFERENCES}

[1] Albeverio, S.; Høegh-Krohn, R.; Fenstad, J.; Lindstrøm, T.: Nonstandard methods in stochastic analysis and mathematical physics. Pure and Applied Mathematics, 122. Academic Press, Inc., Orlando, FL, 1986.

[2] Anderson, M.; Cheeger, J.: $C^{\alpha}$-compactness for manifolds with Ricci curvature and injectivity radius bounded below. J. Differential Geom. 35 (1992), no. $2,265-281$.

[3] Anderson, R. M.: Infinitesimal Methods in Mathematical Economics, Preprint, 2000. See http://www.econ.berkeley.edu/ anderson/Book.pdf

[4] Arkeryd, L.: Intermolecular forces of infinite range and the Boltzmann equation. Arch. Rational Mech. Anal. 77 (1981), no. 1, 11-21.

[5] Arkeryd, L.: Nonstandard analysis. Amer. Math. Monthly 112 (2005), no. 10, 926-928.

[6] Artigue, M.: Analysis, in Advanced Mathematical Thinking (see [173]), Springer-Verlag (1994), p. 172 ("The non-standard analysis revival and its weak impact on education").

[7] Avigad, J.: Weak theories of nonstandard arithmetic and analysis. Reverse mathematics 2001, 19-46, Lect. Notes Log., 21, Assoc. Symbol. Logic, La Jolla, CA, 2005.

[8] Avigad, J.: Response to questionnaire. In Vincent F. Hendricks and Hannes Leitgeb, editors, Philosophy of Mathematics: 5 questions, Automatic / VIP Press, 2007.

[9] Avigad, J.: The metamathematics of ergodic theory. Ann. Pure Appl. Logic 157 (2009), no. 2-3, 64-76.

[10] Avigad, J.; Reck, E.: "Clarifying the nature of the infinite": the development of metamathematics and proof theory. December 11, 2001. Carnegie Mellon Technical Report CMU-PHIL-120.

[11] Bacon, Francis: Novum Organum, 1620.

[12] Barreau, H. and Harthong, J., eds. La mathématique non standard. Histoire. Philosophie. Dossier scientifique. Fondements des Sciences. Editions du Centre National de la Recherche Scientifique (CNRS), Paris, 1989.

[13] Beeson, M.: Foundations of constructive mathematics. Metamathematical studies. Ergebnisse der Mathematik und ihrer Grenzgebiete (3), 6. SpringerVerlag, Berlin, 1985. 
[14] Beeson, M.: Constructive geometry, in Arai, T. (ed.) Proceedings of the Tenth Asian Logic Colloquium, Kobe, Japan, 2008, World Scientific, Singapore, 2009.

[15] Bell, J. L.: A primer of infinitesimal analysis. Second edition. Cambridge University Press, Cambridge, 2008.

[16] Bell, J. L.: Continuity and infinitesimals. Stanford Encyclopedia of philosophy. Revised 20 july 2009.

[17] Bell, J. L.: Le continu cohésif. Intellectica 2009/1, no. 5.

[18] Berkeley, G.: The Analyst, a Discourse Addressed to an Infidel Mathematician (1734).

[19] Bernstein, A. R.; Robinson, A.: Solution of an invariant subspace problem of K. T. Smith and P. R. Halmos, Pacific Journal of Mathematics 16 (1966), 421-431.

[20] Billinge, H.: Applied constructive mathematics: on G. Hellman's "Mathematical constructivism in spacetime". British J. Philos. Sci. 51 (2000), no. 2, 299-318.

[21] Billinge, H.: Did Bishop have a philosophy of mathematics? Philos. Math. (3) 11 (2003), no. 2, 176-194.

[22] Birkhoff, G. (Ed.): Proceedings of the American Academy Workshop on the Evolution of Modern Mathematics. Held at the American Academy of Arts and Sciences, Boston, Mass., Aug. 7-9, 1974. Edited by Garrett Birkhoff, with the assistance of Sue Ann Garwood. With a foreword by John Voss. Historia Math. 2 (1975), no. 4. University of Toronto Press, Toronto, Ont., 1975. pp. i-iv and 425-624.

[23] Bishop, E.: Foundations of constructive analysis. McGraw-Hill Book Co., New York-Toronto, Ont.-London 1967.

[24] Bishop, E.: Mathematics as a numerical language. 1970 Intuitionism and Proof Theory (Proc. Conf., Buffalo, N.Y., 1968) pp. 53-71. North-Holland, Amsterdam.

[25] Bishop, E.: The crisis in contemporary mathematics. Proceedings of the American Academy Workshop on the Evolution of Modern Mathematics (Boston, Mass., 1974). Historia Math. 2 (1975), no. 4, 507-517.

[26] Bishop, E.: Review: H. Jerome Keisler, Elementary calculus, Bull. Amer. Math. Soc. 83 (1977), 205-208.

[27] Bishop, E.: Schizophrenia in contemporary mathematics. In Errett Bishop: reflections on him and his research (San Diego, Calif., 1983), 1-32, Contemp. Math. 39, Amer. Math. Soc., Providence, RI, 1985.

[28] Bishop, E.; Bridges, D.: Constructive analysis. Grundlehren der Mathematischen Wissenschaften [Fundamental Principles of Mathematical Sciences], 279. Springer-Verlag, Berlin, 1985.

[29] Błaszczyk, P.: Nonstandard Analysis from a Philosophical Point of View. In Non-Classical Mathematics 2009 (Hejnice, 18-22 june 2009), pp. 21-24.

[30] Boniface, J.; Schappacher, N.: "Sur le concept de nombre en mathématique": cours inédit de Leopold Kronecker à Berlin (1891). ["On the concept of number in mathematics": Leopold Kronecker's 1891 Berlin lectures] Rev. Histoire Math. 7 (2001), no. 2, 206-275.

[31] Borovik, A.; Katz, M.: Who gave you the Cauchy-Weierstrass tale? The dual history of rigorous calculus. Foundations of Science, 2011, see 
http://dx.doi.org/10.1007/s10699-011-9235-x and http://arxiv.org/abs/1108.2885

[32] Bråting, K.: A new look at E. G. Björling and the Cauchy sum theorem. Arch. Hist. Exact Sci. 61 (2007), no. 5, 519-535.

[33] Bridges, D.: A constructive look at the real number line. In Real numbers, generalizations of the reals, and theories of continua (see 61), 29-92.

[34] Bridges, D.: Constructive mathematics and unbounded operators. Reply to: "Constructive mathematics and quantum mechanics: unbounded operators and the spectral theorem" by G. Hellman. J. Philos. Logic 24 (1995), no. 5, $549-561$.

[35] Bridges, D.: Review of Interview with a constructive mathematician (Math Reviews), 1997, online at http://www.ams.org/mathscinet-getitem?mr=1400617

[36] Bridges, D.: Can constructive mathematics be applied in physics? J. Philos. Logic 28 (1999), no. 5, 439-453.

[37] Burgess, J.: Mathematics and Bleak House. Philosophia Mathematica (3) 12 (2004), 18-36.

[38] Caspers, M.; Heunen, C.; Landsman, N.; Spitters, B.: Intuitionistic quantum logic of an $n$-level system. Found. Physics. See arXiv:0902.3201

[39] Cauchy, A. L.: Cours d'Analyse de L'Ecole Royale Polytechnique. Première Partie. Analyse algébrique. Paris: Imprimérie Royale, 1821. Online at http://books.google.com/books?id=_mYVAAAAQAAJ\&dq=cauchy\&lr=\&source=gbs_navlinks_s

[40] Cleave, J.: Cauchy, convergence and continuity. British J. Philos. Sci. 22 (1971), 27-37.

[41] Colyvan, M.: The indispensability of mathematics. Oxford University Press, Oxford, 2001.

[42] Connes, A.: Noncommutative geometry and reality. J. Math. Phys. 36 (1995), no. 11, 6194-6231.

[43] Corry, L.: Axiomatics, empiricism, and Anschauung in Hilbert's conception of geometry: between arithmetic and general relativity. The architecture of modern mathematics, 133-156, Oxford Univ. Press, Oxford, 2006.

[44] Crowe, M.: Duhem and history and philosophy of mathematics. Pierre Duhem: historian and philosopher of science, Part II. Synthese 83 (1990), no. 3, 431-447.

[45] Crowe, M.: Afterword (1992): a revolution in the historiography of mathematics? In Revolutions in mathematics, 306-316, Oxford Sci. Publ., Oxford Univ. Press, New York, 1992.

[46] Cutland, N.; Kessler, C.; Kopp, E.; Ross, D.: On Cauchy's notion of infinitesimal. British J. Philos. Sci. 39 (1988), no. 3, 375-378.

[47] Dauben, J.: Abraham Robinson and Nonstandard Analysis: History, Philosophy, and Foundations of Mathematics. In William Aspray and Philip Kitcher, eds. History and philosophy of modern mathematics (Minneapolis, MN, 1985), 177-200, Minnesota Stud. Philos. Sci., XI, Univ. Minnesota Press, Minneapolis, MN, 1988. Available online at http://www.mcps.umn.edu/assets/pdf/11_ModernMath.pdf

[48] Dauben, J.: Conceptual revolutions and the history of mathematics: two studies in the growth of knowledge (1984). In Revolutions in mathematics, 49-71, Oxford Sci. Publ., Oxford Univ. Press, New York, 1992. 
[49] Dauben, J.: Appendix (1992): revolutions revisited. pp. 72-82 in Revolutions in mathematics. Edited and with an introduction by Donald Gillies. Oxford Science Publications. The Clarendon Press, Oxford University Press, New York, 1992.

[50] Dauben, J.: Abraham Robinson. The creation of nonstandard analysis. A personal and mathematical odyssey. With a foreword by Benoit B. Mandelbrot. Princeton University Press, Princeton, NJ, 1995.

[51] Dauben, J.: Arguments, logic and proof: mathematics, logic and the infinite. History of mathematics and education: ideas and experiences (Essen, 1992), 113-148, Stud. Wiss. Soz. Bildungsgesch. Math., 11, Vandenhoeck \& Ruprecht, Göttingen, 1996.

[52] Dauben, J.: "Abraham Robinson. 1918-1974," Biographical Memoirs of the National Academy of Sciences 82 (2003), 243-284. Available at the address http://www.nap.edu/html/biomems/arobinson.pdf and http://www.nap.edu/catalog/10683.html

[53] Davies, E. B.: A defence of mathematical pluralism. Philos. Math. (3) 13 (2005), no. 3, 252-276.

[54] Davis, M.: Applied nonstandard analysis. Pure and Applied Mathematics. Wiley-Interscience [John Wiley \& Sons], New York-London-Sydney, 1977. Reprinted: Dover, NY, 2005, see http://store.doverpublications.com/0486442292.html

[55] Davis, M.: Review: J. Donald Monk, Mathematical logic, Bull. Amer. Math. Soc. 83 (1977), 1007-1011.

[56] Davis, M.; Hausner, M.: Book review. The Joy of Infinitesimals. J. Keisler's Elementary Calculus. Mathematical Intelligencer 1 (1978), 168-170.

[57] Diaconescu, R.: Axiom of choice and complementation. Proceedings of the American Mathematical Society 51 (1975), 176-178.

[58] Dossena, R.; Magnani, L.: Mathematics through Diagrams: Microscopes in Non-Standard and Smooth Analysis. Studies in Computational Intelligence (SCI) 64 (2007), 193-213.

[59] Dummett, M.: Elements of intuitionism. Written with the assistance of Roberto Minio. Oxford Logic Guides. Clarendon Press, Oxford, 1977.

[60] Dummett, M.: Elements of intuitionism. Second edition. Oxford Logic Guides, 39. The Clarendon Press, Oxford University Press, New York, 2000.

[61] Ehrlich, P., Ed.: Real numbers, generalizations of the reals, and theories of continua. Edited by Philip Ehrlich. Synthese Library, 242. Kluwer Academic Publishers Group, Dordrecht, 1994.

[62] Ehrlich, P.: The absolute arithmetic continuum and the unification of all numbers great and small. Bulletin of Symbolic Logic, to appear.

[63] Ewing, John: private communication (2009)

[64] Ely, R.: Nonstandard student conceptions about infinitesimals. Journal for Research in Mathematics Education 41 (2010), no. 2, 117-146.

[65] Feferman, S.: What rests on what? The proof-theoretic analysis of mathematics. Philosophy of mathematics (Kirchberg am Wechsel, 1992), 147171, Schriftenreihe Wittgenstein-Ges., 20/I, Hölder-Pichler-Tempsky, Vienna, 1993. 
[66] Feferman, S.: Relationships between constructive, predicative and classical systems of analysis. Proof theory (Roskilde, 1997), 221-236, Synthese Lib., 292, Kluwer Acad. Publ., Dordrecht, 2000.

[67] Feferman, S.: The significance of Weyl's Das Kontinuum. Proof theory (Roskilde, 1997), 179-194, Synthese Lib., 292, Kluwer Acad. Publ., Dordrecht, 2000.

[68] Feferman, S.: Conceptions of the continuum [Le continu mathématique. Nouvelles conceptions, nouveaux enjeux]. Intellectica 51 (2009) 169-189. See also http://math.stanford.edu/ feferman/papers/ConceptContin.pdf

[69] Fernández, E.: The students' take on the epsilon-delta definition of a limit. Primus: Problems, Resources, and Issues in Mathematics Undergraduate Studies, 1935-4053, Volume 14, (2004), no 1, 43-54.

[70] Fraenkel, A.: Einleitung in die Mengenlehre. Dover Publications, New York, N. Y., 1946 [originally published by Springer, Berlin, 1928].

[71] Frege, Gottlob: Begriffsschrift und andere Aufsätze. (German) Zweite Auflage. Mit E. Husserls und H. Scholz' Anmerkungen herausgegeben von Ignacio Angelelli Georg Olms Verlagsbuchhandlung, Hildesheim 1964

[72] Freudenthal, H.: Did Cauchy plagiarise Bolzano? Arch. Hist. Exact Sci. 7 (1971), 375-392.

[73] Gelfand, I.; Kolmogoroff, A.: On rings of continuous functions on topological spaces. (English) [J] C. R. (Dokl.) Acad. Sci. URSS, n. Ser. 22 (1939), 11-15.

[74] Gillies, D. (Ed.): Revolutions in Mathematics. 1992. Oxford University Press, Oxford.

[75] Gillies, D.: The Fregean revolution in logic. In Revolutions in mathematics, 265-305, Oxford Sci. Publ., Oxford Univ. Press, New York, 1992.

[76] Giordano, P.: Fermat reals: Nilpotent infinitesimals and infinite dimensional spaces. arXiv:0907.1872, July 2009.

[77] Giordano, P.: Infinitesimals without logic. Russian Journal of Mathematical Physics, 17(2) (2010), 159-191.

[78] Giordano, P.: The ring of Fermat reals. Advances in Mathematics, 225(4) (2010), 2050-2075.

[79] Giordano, P.; Katz, M.: Two ways of obtaining infinitesimals by refining Cantor's completion of the reals, 2011, see http://arxiv.org/abs/1109.3553

[80] Goldblatt, R.: Lectures on the hyperreals. An introduction to nonstandard analysis. Graduate Texts in Mathematics, 188. Springer-Verlag, New York, 1998.

[81] Goodman, N. D.; Myhill, J.: Choice Implies Excluded Middle. Zeitschrift fur Mathematische Logik und Grundlaaen der Mathematik 24 (1975) 461.

[82] Kreisel, G.: Review: Wittgenstein's Remarks on the Foundations of Mathematics. The British Journal for the Philosophy of Science 9 (1958), no. 34, $135-158$.

[83] Gordon, E. I.; Kusraev, A. G.; Kutateladze, S. S.: Infinitesimal analysis. Updated and revised translation of the 2001 Russian original. Translated by Kutateladze. Mathematics and its Applications, 544. Kluwer Academic Publishers, Dordrecht, 2002.

[84] Grattan-Guinness, I.: Letter. Math. Intelligencer 1 (1978/79), no. 4, 247248. 
[85] Grattan-Guinness, I.: The mathematics of the past: distinguishing its history from our heritage. Historia Mathematica 31 (2004), 163-185.

[86] Grattan-Guinness, I.: Solving Wigner's mystery: the reasonable (though perhaps limited) effectiveness of mathematics in the natural sciences. Math. Intelligencer 30 (2008), no. 3, 7-17.

[87] Gray, J.: The nineteenth-century revolution in mathematical ontology. In Revolutions in mathematics, 226-248, Oxford Sci. Publ., Oxford Univ. Press, New York, 1992.

[88] Gromov, M.: Metric structures for Riemannian and non-Riemannian spaces. Based on the 1981 French original. With appendices by M. Katz, P. Pansu and S. Semmes. Translated from the French by Sean Michael Bates. Reprint of the 2001 English edition. Modern Birkhäuser Classics. Birkhäuser Boston, Inc., Boston, MA, 2007.

[89] Halmos, P.: I want to be a mathematician. An automathography. SpringerVerlag, New York, 1985.

[90] Halmos, P.: Invariant subspaces of polynomially compact operators, Pacific Journal of Mathematics 16 (1966), 433-437.

[91] Hawking, S. W.; Ellis, G. F. R.: The large scale structure of space-time. Cambridge Monographs on Mathematical Physics, No. 1. Cambridge University Press, London-New York, 1973.

[92] Heidegger, M.: Being and time (J. Macquarrie and E. Robinson, trans.) Harper \& Row, New York, 1962.

[93] Hellman, G.: Never Say "Never"! On the Communication Problem between Intuitionism and Classicism in Philosophy of Mathematics. Philosophical Topics 17 (1989), no 2, 47-67.

[94] Hellman, G.: On the Scope and Force of Indispensability Arguments. PSA: Proceedings of the Biennial Meeting of the Philosophy of Science Association, Vol. 1992, Volume Two: Symposia and Invited Papers (1992), 456-464.

[95] Hellman, G.: Constructive Mathematics and Quantum Mechanics: Unbounded Operators and the Spectral Theorem, Journal of Philosophical Logic 12 (1993), 221-248.

[96] Hellman, G.: Gleason's theorem is not constructively provable. J. Philos. Logic 22 (1993), no. 2, 193-203.

[97] Hellman, G.: Quantum mechanical unbounded operators and constructive mathematics - a rejoinder to Bridges. J. Philos. Logic 26 (1997), no. 2, 121127.

[98] Hellman, G.: Mathematical constructivism in spacetime. British J. Philos. Sci. 49 (1998), no. 3, 425-450.

[99] Hewitt, E.: Rings of real-valued continuous functions. I. Trans. Amer. Math. Soc. 64 (1948), 45-99.

[100] Hewitt, E.: So far, so good: my life up to now. The Mathematical Intelligencer 12 (1990), no. 3, 58-63. See http://at.yorku.ca/t/o/p/c/86.dir/hewitt.htm

[101] Heyting, A.: Intuitionism. An introduction. North-Holland Publishing Co., Amsterdam, 1956.

[102] Heijting, A.: Address to Professor A. Robinson. At the occasion of the Brouwer memorial lecture given by Prof. A.Robinson on the 26th April 1973. Nieuw Arch. Wisk. (3) 21 (1973), 134-137. MathScinet Review at 
http://www.ams.org/mathscinet-getitem?mr=434756

[103] Hilbert, D. (1915) Die Grundlagen der Physik, Konigl. Gesell. d. Wiss. Gottingen, Nachr. Math.-Phys. Kl. 395-407.

[104] Hilbert, D. (1919-20), Natur und Mathematisches Erkennen: Vorlesungen, gehalten 1919-1920 in Göttingen. Nach der Ausarbeitung von Paul Bernays (Edited and with an English introduction by David E. Rowe), Basel, Birkhäuser (1992).

[105] Isbell, J. R.: More on the continuity of the real roots of an algebraic equation. Proc. Amer. Math. Soc. 5 (1954), 439.

[106] Ishihara, H.: A constructive version of Banach's inverse mapping theorem. New Zealand J. Math. 23 (1994), no. 1, 71-75.

[107] Kanovei, V.; Shelah, S.: A definable nonstandard model of the reals. J. Symbolic Logic 69 (2004), no. 1, 159-164.

[108] Katz, M.: Systolic geometry and topology. With an appendix by Jake P. Solomon. Mathematical Surveys and Monographs, 137. American Mathematical Society, Providence, RI, 2007.

[109] Katz, K.; Katz, M.: Zooming in on infinitesimal $1-.9$.. in a post-triumvirate era. Educational Studies in Mathematics 74 (2010), no. 3, 259-273. See arXiv:1003.1501.

[110] Katz, K.; Katz, M.: When is .999 . . less than 1? The Montana Mathematics Enthusiast 7 (2010), No. 1, 3-30.

[111] Katz, K.; Katz, M.: A Burgessian critique of nominalistic tendencies in contemporary mathematics and its historiography. Foundations of Science (2011), see http://dx.doi.org/10.1007/s10699-011-9223-1 and http://arxiv.org/abs/1104.0375

[112] Katz, K.; Katz, M.: Cauchy's continuum. Perspectives on Science 19 (2011), no. $4,426-452$.

see http://dx.doi.org/10.1162/POSC_a_00047 and http://arxiv.org/abs/1108.4201

[113] Katz, K.; Katz, M.: Stevin numbers and reality, Foundations of Science, 2011 , see http://dx.doi.org/10.1007/s10699-011-9228-9 and http://arxiv.org/abs/1107.3688

[114] Katz, M.; Tall, D.: The tension between intuitive infinitesimals and formal mathematical analysis. Chapter in Crossroads in the History of Mathematics and Mathematics Education. Bharath Sriraman, Editor. Soon to be available at http://www.infoagepub.com/products/Crossroads-in-the-History-of-Mathematics

[115] Keisler, H. J.: Letter to the editor. Notices Amer. Math. Soc. 24 (1977), 269.

[116] Keisler, H. J.: Elementary Calculus: An Infinitesimal Approach. Second Edition. Prindle, Weber \& Schimidt, Boston, 1986.

[117] Keisler, H. J.: The hyperreal line. Real numbers, generalizations of the reals, and theories of continua, 207-237, Synthese Lib., 242, Kluwer Acad. Publ., Dordrecht, 1994.

[118] Keisler, H. J.: The ultraproduct construction. Proceedings of the Ultramath Conference, Pisa, Italy, 2008. 
[119] Klein, F.: Elementary Mathematics from an Advanced Standpoint. Vol. I. Arithmetic, Algebra, Analysis. Translation by E. R. Hedrick and C. A. Noble [Macmillan, New York, 1932] from the third German edition [Springer, Berlin, 1924]. Originally published as Elementarmathematik vom höheren Standpunkte aus (Leipzig, 1908).

[120] Kohlenbach, U.; Oliva, P.: Proof mining: a systematic way of analyzing proofs in mathematics. Tr. Mat. Inst. Steklova 242 (2003), Mat. Logika i Algebra, 147-175; translation in Proc. Steklov Inst. Math. 2003, no. 3 (242), 136-164.

[121] Kolmogorov, A. N.: Modern debates on the nature of mathematics. With a commentary by V. A. Uspenskii. Reprinted from Nauchae Slovo 1929, no. 6, 41-54. Problemy Peredachi Informatsii 42 (2006), no. 4, 129-141; translation in Probl. Inf. Transm. 42 (2006), no. 4, 379-389.

[122] Komkov, V.: Letter to the editor, Notices Amer. Math. Soc. 24 (1977), no. 5, 269-271.

[123] Kopell, N.; Stolzenberg, G.: Commentary on E. Bishop's talk (Historia Math. 2 (1975), 507-517). Proceedings of the American Academy Workshop on the Evolution of Modern Mathematics (Boston, Mass., 1974). Historia Math. 2 (1975), no. 4, 519-521.

[124] Lakatos, I.: Cauchy and the continuum: the significance of nonstandard analysis for the history and philosophy of mathematics. Math. Intelligencer 1 (1978), no. 3, 151-161 (originally published in 1966).

[125] Lawvere, F. W.: Toward the description in a smooth topos of the dynamically possible motions and deformations of a continuous body. Third Colloquium on Categories (Amiens, 1980), Part I. Cahiers Topologie Géom. Différentielle 21 (1980), no. 4, 377-392.

[126] Lightstone, A. H.: Infinitesimals. Amer. Math. Monthly 79 (1972), 242-251.

[127] Łoś, J.: Quelques remarques, théorèmes et problèmes sur les classes définissables d'algèbres. In Mathematical interpretation of formal systems, 98113, North-Holland Publishing Co., Amsterdam, 1955.

[128] Maddy, P.: The roots of contemporary Platonism. J. Symbolic Logic 54 (1989), no. 4, 1121-1144.

[129] Magnani, L.; Dossena, R.: Perceiving the infinite and the infinitesimal world: unveiling and optical diagrams in mathematics. Foundations of Science $\mathbf{1 0}$ (2005), no. 1, 7-23.

[130] Malet, A.: Renaissance notions of number and magnitude. Historia Mathematicae 33 (2006), no. 1, 63-81.

[131] Manning, Kenneth: private communication, july 2009.

[132] McCarthy, T.: Review of E. Bishop's essay "The crisis in contemporary mathematics" (item 25] above), 1975, online at http://www.ams.org/mathscinet-getitem? $\mathrm{mr}=498014$

[133] Meschkowski, H.: Aus den Briefbuchern Georg Cantors. Archive for History of Exact Sciences 2 (1965), 503-519.

[134] Mormann, T.: Idealization in Cassirer's philosophy of mathematics. Philos. Math. (3) 16 (2008), no. 2, 151-181.

[135] Myers, S. B.: Riemannian manifolds with positive mean curvature. Duke Math. J. 8 (1941), 401-404. 
[136] Naets, J.: How to define a number? A general epistemological account of Simon Stevin's art of defining. Topoi 29 (2010), no. 1, 77-86.

[137] Novikov, S. P.: The second half of the 20th century and its results: the crisis of the society of physicists and mathematicians in Russia and in the West. (Russian) Istor.-Mat. Issled. (2) No. 7(42) (2002), 326-356, 369.

[138] Novikov, S. P.: The second half of the 20th century and its conclusion: crisis in the physics and mathematics community in Russia and in the West. Amer. Math. Soc. Transl. Ser. 2, 212, Geometry, topology, and mathematical physics, 1-24, Amer. Math. Soc., Providence, RI, 2004. (Translated from Istor.-Mat. Issled. (2) No. 7(42) (2002), 326-356, 369; by A. Sossinsky.)

[139] Palmgren, E.: Developments in constructive nonstandard analysis. Bull. Symbolic Logic 4 (1998), no. 3, 233-272.

[140] Penrose, R.: Techniques of differential topology in relativity. Conference Board of the Mathematical Sciences Regional Conference Series in Applied Mathematics, No. 7. Society for Industrial and Applied Mathematics, Philadelphia, Pa., 1972.

[141] Pourciau, B.: The education of a pure mathematician. Amer. Math. Monthly 106 (1999), no. 8, 720-732.

[142] Pourciau, B.: Intuitionism as a (failed) Kuhnian revolution in mathematics. Stud. Hist. Philos. Sci. 31A (2000), no. 2, 297-329.

[143] Putnam, H.: Wittgenstein and the Real Numbers. Wittgenstein and the Moral Life, ed. Alice Crary (Cambridge, MA: MIT Press, 2007), 235-250.

[144] Richman, F.: Confessions of a formalist, Platonist intuitionist. April 9, 1994. See http://math.fau.edu/Richman/HTML/Confess.htm

[145] Richman, F.: Interview with a constructive mathematician. Modern Logic 6 (1996), no. 3, 247-271.

[146] Robinson, A.: On the metamathematics of algebra. Studies in Logic and the Foundations of Mathematics. North-Holland Publishing Co., Amsterdam, 1951.

[147] Robinson, A.: Non-standard analysis. Nederl. Akad. Wetensch. Proc. Ser. A 64 = Indag. Math. textbf23 (1961), 432-440 [reprinted in Selected Works, see item [151], pp. 3-11]

[148] Robinson, A.: Non-standard analysis. North-Holland Publishing Co., Amsterdam 1966.

[149] Robinson, A.: "Reviews: Foundations of Constructive Analysis". Amer. Math. Monthly 75 (1968), no. 8, 920-921.

[150] Robinson, A.: Standard and nonstandard number systems. The Brouwer memorial lecture 1973, Leiden, April 26, 1973. Nieuw Arch. Wisk. (3) 21 (1973), 115-133.

[151] Robinson, A.: Selected papers of Abraham Robinson. Vol. II. Nonstandard analysis and philosophy. Edited and with introductions by W. A. J. Luxemburg and S. Körner. Yale University Press, New Haven, Conn., 1979.

[152] Robinson, A.: Non-standard analysis. Reprint of the second (1974) edition. With a foreword by Wilhelmus A. J. Luxemburg. Princeton Landmarks in Mathematics. Princeton University Press, Princeton, NJ, 1996.

[153] Roh, K.H.: Students' images and their understanding of definitions of the limit of a sequence. Educational Studies in Mathematics 69 (2008), 217-233. 
[154] Rosenblatt, M., Editor. Errett Bishop: reflections on him and his research. Proceedings of the memorial meeting for Errett Bishop held at the University of California, San Diego, Calif., September 24, 1983. Contemporary Mathematics, 39. American Mathematical Society, Providence, RI, 1985.

[155] Ross, D. A.: A nonstandard proof of a lemma from constructive measure theory. MLQ Math. Log. Q. 52 (2006), no. 5, 494-497.

[156] Rust, H.: Operational Semantics for Timed Systems. Lecture Notes in Computer Science 3456 (2005), 23-29, DOI: 10.1007/978-3-540-32008-1_4.

[157] Schubring, G.: Conflicts between generalization, rigor, and intuition. Number concepts underlying the development of analysis in 17-19th Century France and Germany. Sources and Studies in the History of Mathematics and Physical Sciences. Springer-Verlag, New York, 2005.

[158] Schuster, P.; Berger, U.; Osswald, H., eds: Reuniting the antipodesconstructive and nonstandard views of the continuum. Proceedings of the symposium held in Venice, May 16-22, 1999. Synthese Library, 306. Kluwer Academic Publishers, Dordrecht, 2001.

[159] Sfard, A: Reification as the birth of metaphor. For the Learning of Mathematics, 14(1) (1994), 44-55.

[160] Shapiro, S.: Why anti-realists and classical mathematicians cannot get along. Topoi 20 (2001), no. 1, 53-63.

[161] Sherry, D.: The wake of Berkeley's Analyst: rigor mathematicae? Stud. Hist. Philos. Sci. 18 (1987), no. 4, 455-480.

[162] Smith, P.: Review of La mathématique non standard (item [12] above); online at http://www.ams.org/mathscinet-getitem?mr=1145982

[163] Stewart, I.: From here to infinity. A retitled and revised edition of The problems of mathematics [Oxford Univ. Press, New York, 1992]. With a foreword by James Joseph Sylvester. The Clarendon Press, Oxford University Press, New York, 1996.

[164] Stolzenberg, G.: Review: Foundations of constructive analysis by Errett Bishop. Bull. Amer. Math. Soc. 76 (1970), 301-323.

[165] Stolzenberg, G.: Can an Inquiry into the Foundations of Mathematics Tell Us Anything Interesting about Mind? In Psychology and Biology of Language and Thought: Essays in Honor of Eric Lenneberg. Academic Press, New York, 1978, pp. 221-269.

[166] Stolzenberg, G.: Letter to the editor. Notices Amer. Math. Soc. 25 (1978), 242.

[167] Stolzenberg, G.: Reading and relativism. An introduction to the science wars. In Ashman, K.; Baringer, P., editors: After the science wars. ebrary, Inc., 2001.

[168] Stroyan, K.: Uniform continuity and rates of growth of meromorphic functions. Contributions to non-standard analysis (Sympos., Oberwolfach, 1970), pp. 47-64. Studies in Logic and Foundations of Math., Vol. 69, NorthHolland, Amsterdam, 1972.

[169] Sullivan, K.: The Teaching of Elementary Calculus Using the Nonstandard Analysis Approach, Amer. Math. Monthly 83 (1976), 370-375.

[170] Tait, W.: Against intuitionism: constructive mathematics is part of classical mathematics. J. Philos. Logic 12 (1983), no. 2, 173-195. 
[171] Tait, W.: Beyond the axioms: the question of objectivity in mathematics. The George Boolos Memorial Symposium, II (Notre Dame, IN, 1998). Philos. Math. (3) 9 (2001), no. 1, 21-36.

[172] Tall, D: Looking at graphs through infinitesimal microscopes, windows and telescopes, The Mathematical Gazette 64 (1980), 22-49.

[173] Tall, D.: Advanced mathematical thinking. Edited by David Tall. Mathematics Education Library, 11. Kluwer Academic Publishers Group, Dordrecht, 1991.

[174] Tall D.: Natural and Formal Infinities. Educational Studies in Mathematics 48 (2001), Numbers 2-3, 199-238.

[175] Tall, D.: Dynamic mathematics and the blending of knowledge structures in the calculus. Transforming Mathematics Education through the use of Dynamic Mathematics, ZDM 41 (june 2009), no. 4, 481-492.

[176] A. Tarski, Une contribution à la théorie de la mesure, Fundamenta Mathematicae 15 (1930), 42-50.

[177] Taylor, M.: Anderson-Cheeger limits of smooth Riemannian manifolds, and other Gromov-Hausdorff limits. J. Geom. Anal. 17 (2007), no. 2, 365-374.

[178] Tennant, N.: The Law of Excluded Middle is Synthetic A Priori, If Valid, Philosophical Topics 24 (1996), 205-229.

[179] Tennant, N.: Logic, Mathematics, and the natural sciences. In Gabbay, D.; Thagard, P.; Woods, J., eds.: Handbook of the philosophy of science. Elsevier, 2006, pp. 1145-1161.

[180] Thomas, G.; Finney, R.: Calculus and analytic geometry. Fifth edition. Addison-Wesley, 1979.

[181] Troelstra, A.; van Dalen, D.: Constructivism in mathematics. Vol. I. An introduction. Studies in Logic and the Foundations of Mathematics, 121. North-Holland Publishing Co., Amsterdam, 1988.

[182] van Rootselaar, B.: Review of item [23] above. See http://www.ams.org/mathscinet-getitem? $\mathrm{mr}=221878$

[183] Wagon, S.: The Banach-Tarski paradox. With a foreword by Jan Mycielski. Corrected reprint of the 1985 original. Cambridge University Press, Cambridge, 1993.

[184] Wald, R.: General relativity. University of Chicago Press, Chicago, IL, 1984.

[185] Warschawski, S. E.: Errett Bishop - in memoriam. Errett Bishop: reflections on him and his research (San Diego, Calif., 1983), 33-39, Contemp. Math., 39, Amer. Math. Soc., Providence, RI, 1985.

[186] Weir, A.: Dummett on meaning and classical logic. Mind textbf95 (1986), no. $380,465-477$.

[187] Weller, K.; Arnon, I.; Dubinsky, E.: Preservice Teachers' Understanding of the Relation Between a Fraction or Integer and Its Decimal Expansion. Canadian Journal of Science, Mathematics and Technology Education, 19424051, Volume 9 (2009), no. 1, 5-28.

[188] Westerståhl, D.: Proofs instead of meaning explanations: understanding classical vs intuitionistic mathematics from the outside. Deduction, computation, experiment, 175-194, Springer Italia, Milan, 2008.

[189] Weyl, H.: Über die neue Grundlagenkrise der Mathematik. (Vorträge, gehalten im mathematischen Kolloquium Zürich.) (German) [J] Math. Zeitschr. 10 (1921), 39-79. 
[190] Weyl, H.: Philosophy of Mathematics and the Natural Sciences, Princeton (1949).

[191] Weyl, H.: The Future of Mathematics. The American Mathematical Monthly $\mathbf{5 8}$ (1951), no. 8, 523-553.

[192] Yau, S.-T.; Nadis, S.: The shape of inner space. String theory and the geometry of the universe's hidden dimensions. Basic Books, New York, 2010. 
Karin Usadi Katz has taught mathematics at Michlelet Banot Lustig, Ramat Gan, Israel. Two of her joint studies with Mikhail Katz were published in Foundations of Science: "A Burgessian critique of nominalistic tendencies in contemporary mathematics and its historiography" and "Stevin numbers and reality", online respectively at

http://dx.doi.org/10.1007/s10699-011-9223-1 and at

http://dx.doi.org/10.1007/s10699-011-9228-9

A joint study with Mikhail Katz entitled "Cauchy's continuum" is due to appear in Perspectives on Science 2011, see http://arxiv.org/abs/1108.4201

Mikhail G. Katz is Professor of Mathematics at Bar Ilan University, Ramat Gan, Israel. Two of his joint studies with Karin Katz were published in Foundations of Science: "A Burgessian critique of nominalistic tendencies in contemporary mathematics and its historiography" and "Stevin numbers and reality", online respectively at

http://dx.doi.org/10.1007/s10699-011-9223-1 and at http://dx.doi.org/10.1007/s10699-011-9228-9

A joint study with Karin Katz entitled "Cauchy's continuum" is due to appear in Perspectives on Science, 2011, see http://arxiv.org/abs/1108.4201

A joint study with Alexandre Borovik entitled "Who gave you the Cauchy-Weierstrass tale? The dual history of rigorous calculus" appeared in Foundations of Science, online at http://dx.doi.org/10.1007/s10699-011-9235-x

A joint study with David Tall, entitled "The tension between intuitive infinitesimals and formal mathematical analysis", is due to appear as a chapter in a book edited by Bharath Sriraman, see http://www.infoagepub.com/products/Crossroads-in-the-History-of-Mathematics

Department of Mathematics, Bar Ilan University, Ramat Gan 52900 ISRAEL

E-mail address: katzmik ' 'at', macs.biu.ac.il 